\title{
Das Globalisierungsskript der Europäischen Union und seine Unterstützung bei den Bürgerinnen und Bürgern in 15 Mitgliedsländern der EU.
}

Jürgen Gerhards \& Philipp Hessel

Berliner Studien zur Soziologie Europas

Nr. 14

Mai 2008

Freie Universität Berlin, Institut für Soziologie, Garystraße 55, D-14195 Berlin 
Die „Berliner Studien zur Soziologie Europas“ des Lehrstuhls für Makrosoziologie der Freien Universität Berlin verstehen sich als ein Ort zur Vorpublikation von Beiträgen, die später in Fachzeitschriften und Sammelbänden veröffentlicht werden sollen. Die Beiträge sollen helfen, eine Soziologie Europas zu profilieren; sie stehen auch im Kontext des Master-Studiengangs „Soziologie - Europäische Gesellschaften“.

Gegenstand der Reihe sind Beiträge zur Analyse der Herausbildung einer europäischen Gesellschaftsstruktur und -kultur, vergleichende Analysen, die die Unterschiede und Gemeinsamkeiten zwischen verschiedenen europäischen Gesellschaften thematisieren, sowie theoretische Versuche einer Soziologie Europas.

Ziel der Reihe ist es, durch die frühe Verbreitung dieser Arbeiten den wissenschaftlichen Gedankenaustausch zu fördern. Die Beiträge sind nur über das Internet als pdf-Datei zu beziehen.

Zitationsweise: BSSE-Arbeitspapier Nr. 14. Berlin: Freie Universität Berlin.

Dieser Artikel erscheint in: „Berliner Journal für Soziologie“. 


\section{Abstract}

Auf der Basis einer Interpretation von Gesetzestexten und öffentlichen Verlautbarungen rekonstruieren wir in einem ersten Schritt die Vorstellungen der Europäischen Union von einer globalisierten Wirtschaft. Die EU verfolgt mit ihren Wirtschaftsvorstellungen und deren Implementierung durch konkrete Politiken das Ziel, Wachstum, Fortschritt und Prosperität für alle Bürger der Mitgliedsländer und für alle Weltbürger zu erzeugen. Dieses Ziel sieht die EU am besten erreichbar, wenn Wettbewerb und die Öffnung von Märkten institutionalisiert werden. Dabei transportiert sie ihre Vorstellungen und Erfahrungen, die im Kontext der Herstellung eines europäischen Wirtschaftsraumes entwickelt wurden auf die Weltgesellschaft insgesamt.

$\mathrm{Ob}$ diese Sichtweise von den Bürgerinnen und Bürgern der EU geteilt wird, haben wir im zweiten Schritt auf der Grundlage einer Auswertung des Eurobarometers für 15 Länder der EU geprüft. Das Ergebnis ist überraschend: 62\% der befragten EUBürger unterstützen die Entwicklung einer ökonomischen Globalisierung durch Marktliberalisierung. Bis auf Griechenland gibt es in allen Ländern eine Mehrheit von Personen, die dem Prozess der Globalisierung positiv gegenüber eingestellt sind. Zugleich fallen die Unterstützungsraten zwischen den Ländern und innerhalb der Länder recht unterschiedlich aus.

Zur Erklärung dieser Unterschiede sind wir von der Annahme ausgegangen, dass diejenigen, die durch Globalisierungsprozesse benachteiligt werden, sich eher gegen den Globalisierungsprozess aussprechen als diejenigen, für die dies nicht gilt. „Benachteiligung" kann sich in drei Dimensionen manifestieren: in der subjektiven Interpretation von positiven und negativen Folgen von Globalisierungsprozessen, in den Nachteilen, die sich aus der objektiven sozialstrukturellen Lage des Individuums ergeben und aus den Nachteilen, die aus der ökonomischen Situation des Landes resultieren.

Auch unsere Kausalanalyse kann mit einem überraschenden Befund aufwarten. Zwar können die Einstellungen der Bürger zur Globalisierung recht gut erklärt werden, allerdings spielen die für Soziologen im Zentrum stehenden Variablen dabei fast keine Rolle. Weder die makroökonomische Situation des Landes, noch die sozialstrukturelle Lage des Interviewten haben einen nennenswerten Effekt auf deren Globalisierungseinstellungen. Die Haltung zur Globalisierung wird fast ausschließlich bestimmt durch den subjektiv definierten Globalisierungsnutzen; dieser ist selbst nicht mehr rückgekoppelt an die objektive Lage des Individuums oder des Landes, in dem das Individuum lebt. 


\section{Einleitung 1}

"Globalisierung“ ist sicherlich eines der am meisten benutzten Modewörter der letzten zehn Jahre zur Beschreibung des Strukturwandels gegenwärtiger Gesellschaften. Die Diskussion politischer Reaktionsmöglichkeiten auf Globalisierungsprozesse steht zudem ganz oben auf der politischen Agenda von fast allen nationalen wie internationalen Akteuren. Dies gilt auch für die Europäische Union. So steht die LissabonStrategie, welche die EU wieder auf Wettbewerbskurs bringen soll, „[...] der in einer modernen globalisierten Wirtschaft Garant für Wachstum und Arbeitsplätze ist [...]“ (Kommission der Europäischen Gemeinschaften 2005), ganz im Zeichen der Globalisierung. Auch auf der informellen Tagung in Hampton Court im Oktober 2005 hatten sich die Staats- und Regierungschefs mit den Herausforderungen der Globalisierung in den Bereichen Innovation, Energie, Migration, Bildung und Demografie auseinander gesetzt (Kommission der Europäischen Gemeinschaften 2005). Auf der Frühjahrstagung des Europäischen Rates 2006 wurde weiterhin beschlossen, die Arbeiten im Rahmen der erneuerten Lissabon-Strategie für Wachstum und Beschäftigung zu intensivieren und dadurch die europäische Wirtschaft fit für die Herausforderungen der Globalisierung zu machen. Dabei versteht sich die Europäische Union selbst als einen der zentralen Akteure, der den Prozess der Globalisierung aktiv zum Wohl der Mitgliedsländer und ihrer Bürger mitgestalten will: „Bei der Europäischen Union müssen alle Fäden der Globalisierungsdebatte zusammenlaufen. Sie muss eine gemeinsame Antwort auf die schwierigste aller Herausforderungen bieten, nämlich den Bürgern deutlich zu machen, dass die Europäer nur mit der EU in der Lage sind, die Globalisierung mit zu gestalten." (Kommission der Europäischen Gemeinschaften 2007c). Insbesondere die Europäische Kommission verweist zunehmend auf die ökonomischen Zwänge der Globalisierung, um spezifische Reformen zu rechtfertigen (Hay und Rosamond 2002).

Während die EU ein positives Bild vom Prozess der Globalisierung zeichnet und dabei auf die positiven Effekte einer globalen Marktöffnung verweist, mag die Einschätzung der Bürger ambivalenter ausfallen. Für die Einen kann der Prozess der Globalisierung neue Möglichkeiten und Chancen eröffnen und mit positiven Erwartungen verbunden sein. Für Andere mag der Prozess eher mit Verlustängsten und Schließungsreaktionen verknüpft sein. Sie sehen Arbeitsplätze bedroht, fühlen sich von Migrationsbewegungen in ihrer nationalen Identität herausgefordert oder befürchten eine Schwächung „ihres“, sie schützenden Nationalstaates.

Wir werden in diesem Artikel in einem ersten Schritt auf der Basis einer Interpretation von Gesetzestexten und von öffentlichen Verlautbarungen der Institutionen der EU genauer untersuchen, welche Globalisierungsvorstellungen die EU entwickelt hat und welche Globalisierungspolitik sie betreibt. Wir bezeichnen diese Vor-

${ }^{1}$ Wir bedanken uns bei David Glowsky, Silke Hans, Sylvia Kämpfer, Ulrich Kohler, Jochen Roose und Joachim Schild für hilfreiche Kommentare. 
stellungen als das Globalisierungsskript der EU. Auch wenn sich die Globalisierungsvorstellungen der EU auf mehrere Politikfelder beziehen (Wirtschaft, Politik, Umwelt, Soziales), so stehen doch Überlegungen im Vordergrund, die sich auf eine globalisierte Wirtschaft beziehen. Wir werden unsere Ausführungen entsprechend auf diese Dimension konzentrieren.

Im zweiten Schritt (Kapitel 3) untersuchen wir dann, ob und in welchem Maße die Bürger der Mitgliedsländer der EU den Globalisierungsoptimismus der EU teilen. Grundlage unserer empirischen Analyse bildet eine Sekundäranalyse eines Eurobarometers (Flash EB 151b/2003 „Globalisation“) aus dem Jahr 2003. Die Umfrage wurde vor der so genannten Osterweiterung der EU durchgeführt; entsprechend beschränken sich unsere Analysen auf 15 Mitgliedsländer der EU. Der Datensatz bietet die Möglichkeit, die Haltungen der Bürger zur ökonomischen Globalisierung zu operationalisieren.

Wir gehen dabei von der Annahme aus, dass die Unterstützung des im Handeln der EU institutionalisierten Globalisierungsskripts durch die Bürger nicht unerheblich für die Legitimität der EU-Politiken ist. Eine Ablehnung der Globalisierungspolitik der EU kann erhebliche Legitimationseinbußen nach sich ziehen. So wurde z.B. der Diskurs über die EU-Verfassung in Frankreich in hohem Maße vom Thema Globalisierung überlagert (Schild 2005). Zudem scheint für einige Akteure eine Opposition gegen Globalisierungsprozesse gleichbedeutend mit einer Opposition gegenüber der EU zu sein, so dass man von einer diskursiven Überlagerung der beiden Themenbereiche sprechen kann (Rosamond 2002: 2). Gerade nationalistische Bewegungen und Parteien, die sich als Fürsprecher der Bewahrung nationaler Identität und Souveränität profilieren, sind häufig gleichermaßen Globalisierungs- und EUKritiker (vgl. Kriesi et al. 2006).

Die deskriptiven Befunde werden zeigen, dass rund 62\% der befragten EU-Bürger den Prozess der Globalisierung grundsätzlich unterstützen. Das ist eine überraschend hohe Unterstützungsquote, bedenkt man die Skepsis, mit der ökonomische Globalisierungsprozesse in der massenmedialen Öffentlichkeit kommentiert werden. Zugleich zeigen sich aber deutliche Unterschiede im Unterstützungsniveau des EUSkripts zwischen den verschiedenen Ländern und innerhalb der Länder. Wir werden entsprechend im Kapitel 4 versuchen, die Unterschiede in den Einstellungen zur Globalisierung zu erklären. Dazu greifen wir auf ein allgemeines Theorem zurück, das wir durch drei Bestimmungsfaktoren weiter spezifizieren. Wir gehen davon aus, dass diejenigen, die durch Globalisierungsprozesse benachteiligt werden sich eher gegen den Globalisierungsprozess aussprechen als diejenigen, für die dies nicht gilt. Wir werden zum einen prüfen, in welchem Maße Einstellungen gegenüber Globalisierungsprozessen durch die vom Befragten erwarteten negativen bzw. positiven Folgen dieses Prozesses bestimmt werden. Dabei unterscheiden wir zwischen individuellen ökonomischen Folgen sowie Folgen für die Volkswirtschaft eines Landes. Wir werden im zweiten Schritt prüfen, in welchem Maße die sozialstrukturelle Lage 
des Befragten (Berufsposition und Bildung) die Einstellungen zur Globalisierung beeinflusst. Schließlich gehen wir drittens der Frage nach, in welchem Maße die makroökonomische Situation des Landes, in dem der Befragte lebt, seine Einstellungen zur Globalisierung beeinflusst. Wir vermuten, dass eine hohe Arbeitslosenrate in einem Land und eine geringe Marktöffnung eines Landes zu einer verstärkten Ablehnung von Globalisierungsprozessen führen et vice versa.

Die Fragestellung und der Aufbau des Artikels folgen anderen Analysen, die wir durchgeführt haben. Wir haben für unterschiedliche Politikbereiche der EU genauer beschrieben, welche Vorstellungen der inhaltlichen Ausgestaltung die EU jeweils entwickelt hat (Religion, Familie und Geschlechterrollen, Demokratie und Zivilgesellschaft, Wirtschaft, Umwelt etc.) und in welchem Maße diese Vorstellungen von den Bürgern unterstützt werden (vgl. z.B. Gerhards und Hölscher 2003; Gerhards 2007; Hölscher 2006; Gerhards und Lengfeld 2006). In diesem Beitrag knüpfen wir an diese Analysen an und ergänzen sie um eine Analyse der Globalisierungsvorstellungen der EU und deren Unterstützung durch die Bürger.

\section{Das Globalisierungsskript der Europäischen Union}

Vertreter des Neoinstitutionalismus interpretieren Institutionen im Allgemeinen und die EU insbesondere als Träger von Ideen und Skripten (Stone Sweet et al. 2001). Theoretisch und empirisch wurde das Skript-Konzept am prominentesten von dem Soziologen John Meyer ausgearbeitet. Ausgangspunkt seiner Studien, insbesondere über die Diffusion von Kindheitsrechten und Bildungssystemen, war die Beobachtung, dass sich in einer wachsenden Anzahl von Ländern der Welt ähnliche Vorstellungen durchsetzen und politisch implementiert werden. Die Erklärung für diese Entwicklung sieht Meyer darin, dass internationale Organisationen normative Vorstellungen bezüglich verschiedener Politikbereiche formulieren und diese durchzusetzen versuchen (Meyer et al. 1997; Meyer et al. 1985; Meyer 2000). Die EU ist eine der internationalen Institutionen, die Träger von Vorstellungen einer wünschenswerten Gesellschaft ist (dazu genauer Gerhards 2007; Wobbe 2001; Wobbe und Biermann 2007). Sie ist zudem eine Organisation, die mit entsprechender Autorität und Sanktionsgewalt ausgestattet ist, ihre Vorstellungen gegenüber den Mitgliedsländern auch durchsetzen zu können.

$\mathrm{Zu}$ den Vorstellungen einer idealen europäischen Gesellschaft gehören auch Annahmen über die Vor- und Nachteile von Globalisierungsprozessen und die Rolle, die Europa in diesem Prozess spielen soll. Das Globalisierungsskript der EU weist verschiedene Dimensionen auf, wobei die ökonomische Dimension die dominante Dimension des Globalisierungsskriptes darstellt. Wir werden uns auf eine Beschreibung dieser Dimension in erster Linie konzentrieren.

Die Vorstellungen der EU bezüglich einer globalisierten Wirtschaft sind unmittelbar abgeleitet aus den Wirtschaftsvorstellungen, die die EU für ihren eigenen - den europäischen - Wirtschaftsraum entwickelt hat. Diese hat Michael Hölscher in seiner 
Dissertation genauer analysiert (Hölscher 2006). Die EU ist als Wirtschaftgemeinschaft entstanden, deren vorderstes Ziel die Schaffung eines gemeinsamen Binnenmarktes war. Bereits in den 1957 unterzeichneten Römischen Verträgen wurde das ehrgeizige Ziel der Schaffung eines gemeinsamen Marktes formuliert. Dies beinhaltete die Bildung einer Zollunion in Form eines Abbaus tarifärer Handelshemmnisse sowie die Einigung auf einen gemeinsamen Außenzoll. Zur endgültigen Durchsetzung der bereits im EWG-Vertrag festgeschriebenen „vier Freiheiten“ (der Freiheit von Gütern, Dienstleistungen, Personen und Kapital) und damit zur Verwirklichung des europäischen Binnenmarktes bedurfte es jedoch einer erneuten Anstrengung in der Mitte der 1980er Jahre, welche schließlich im 1992 unterzeichneten Vertrag von Maastricht ihren Abschluss fand. Heute ist der Handel zwischen den Mitgliedstaaten grundsätzlich keinen Beschränkungen unterworfen.

Die Herstellung von Märkten und die Erzeugung von Wettbewerb sind keine „Endziele“, sondern Mittel für einen bestimmen Zweck. Die EU verfolgt mit ihren Wirtschaftsvorstellungen vor allem ein zentrales „Megaziel“. Sie möchte die ökonomische Wohlfahrt aller Bürger der Mitgliedsländer erhöhen. Die Herstellung von Wettbewerb und die Liberalisierung wettbewerbsbehindernder Reglementierungen verbindet die EU mit der wirtschaftspolitischen Hoffnung der Erzeugung von Wachstum, Fortschritt und Prosperität. Diese, auf den innereuropäischen Raum bezogene Wirtschaftsphilosophie wird nun von der EU auch auf die Weltgesellschaft übertragen. Vor allem die Europäische Kommission als Trägerin des EUGlobalisierungsskripts verfolgt die Idee, dass Marktöffnung und Handel die beste Quelle für Dynamisierung, Wandel und wirtschaftliches Wachstum sind (Kommission der Europäischen Gemeinschaften 2007a: 2). Besonders deutlich spiegelt sich diese Vorstellung in folgendem Zitat aus der Anlage „Erklärung der EU zur Globalisierung“ der Schlussfolgerungen des Vorsitzes des Europäischen Rates vom Dezember 2007 wider. Dort heißt es: „Die Europäische Union wird auf immer offenere Märkte drängen, die zu gegenseitigem Vorteil gereichen sollen." (Europäischer Rat 2007). Weiterhin, so die Auffassung der Kommission, zwingt die Globalisierung (europäische) Unternehmen dazu, ihre Effizienz sowie ihre Innovations- und Wettbewerbsfähigkeit zu erhöhen, was Europa für internationale Investoren attraktiver mache. Entsprechend heißt es etwa in einer Mitteilung der Kommission: „Ein offener europäischer Markt ist unverzichtbar für Wachstum und Schaffung von Arbeitsplätzen in Europa und für unsere internationale Wettbewerbsfähigkeit. Wenn wir offen sind für globalen Handel und Investitionen, können wir auch die Vorteile eines funktionierenden Binnenmarktes besser nutzen." (Kommission der Europäischen Gemeinschaften 2006).

Die Institutionalisierung des Gemeinsamen Marktes und die Öffnung der EU für einen globalen Welthandel bringt aus der Perspektive der EU aber nicht nur Vorteile für die Unternehmungen und die Wirtschaft, sondern auch für die Bürger: „Ziel ist ein Europa, [...] in dem Verbraucher und Unternehmen die Vorteile des Binnen- 
markts in vollem Umfang wahrnehmen." (Kommission der Europäischen Gemeinschaften 2004: 4) Die EU geht dabei davon aus, dass die Bürger die Globalisierung vor allem entlang dieser Nutzendimension beurteilen werden: „Wachstum und Arbeitsplätze und die damit verbundenen Chancen sind das Kernstück der Kommissionsagenda für Europa. Sie sind eine wesentliche Voraussetzung für wirtschaftlichen Wohlstand, soziale Gerechtigkeit und nachhaltige Entwicklung und dafür, dass die Europäer die Globalisierung bewältigen können. Außerdem werden die Bürger vor allem anhand dieser Kriterien beurteilen, ob Europa für ihren Alltag wirklich ein Gewinn ist." (Kommission der Europäischen Gemeinschaften 2006).

Insbesondere die im Jahr 2000 in Lissabon vereinbarte Reformstrategie, die Europa zur wettbewerbsfähigsten wissensbasierten Gesellschaft der Welt führen soll, „,...] mit mehr und besseren Arbeitsplätzen und einem stärkeren sozialen Zusammenhalt [...]“ (Kommission der Europäischen Gemeinschaften 2006: 6), bildet die Grundlage für die konkreten Reaktionen der EU auf die Globalisierungsfolgen für Unternehmen, Arbeitsplätze und Bürger in Europa. Dabei sollen Maßnahmen in folgenden Bereichen durchgeführt werden: Wachstum und Beschäftigung, nachhaltiges Europa, Steuerung der Migration, Bürgerinnen und Bürger im Mittelpunkt sowie Europa als Partner in der Welt (Kommission der Europäischen Gemeinschaften 2007d).

Im Hinblick auf die externe Dimension des EU-Handels ist zunächst festzuhalten, dass die EU die größte Handelsmacht ist und rund ein Fünftel des gesamten Welthandels auf sich vereint (Fligstein und Merand 2001: 24). Die Ziele der gemeinsamen Handelspolitik der EU sind dabei in Art. 131 des EG-Vertrages niedergelegt. Gemäß Art. 131 EGV beabsichtigen die Mitgliedstaaten im gemeinsamen Interesse zur harmonischen Entwicklung des Welthandels, zur schrittweisen Beseitigung der Beschränkungen im internationalen Handelsverkehr und zum Abbau der Zollschranken beizutragen. Mit Hilfe der gemeinsamen Handelspolitik als vergemeinschaftetem Politikbereich hat die EU sowohl in bi- und multilateralen Verhandlungen einen maßgeblichen Beitrag zur Reduzierung sowie Beseitigung der in Art. 131 EGV genannten tarifären und nicht-tarifären Handelshemmnissen geleistet (Meunier und Nicolaïdis 2005: 26). Die Europäische Kommission vertritt, in Analogie zu den Erfahrungen und Regeln des gemeinsamen Marktes eine Politik, welche auf die Schaffung eines auf Regeln basierenden Welthandels abzielt. Im Mittelpunkt stehen dabei die Zusammenarbeit bei der Rechtsetzung, die Konvergenz der Normen und die Gleichwertigkeit der Vorschriften (Kommission der Europäischen Gemeinschaften 2004: 6), wobei die EU international eine Vorreiterrolle einnimmt. Entsprechend haben durch den Binnenmarkt geprägte Vorschriften vielfach als Vorlage für weltweite Standards gedient. Neben einer von der EU propagierten Beseitigung bzw. eines Abbaus von Zöllen setzt sich die EU vor allem auch für den weiteren Abbau nichttarifärer Handelsschranken sowie „[...] international vereinbarte Regeln in Bezug auf fairen Wettbewerb und Schutz der Rechte an geistigem Eigentum“ (Europäischer Rat 2007) ein. 
Die Philosophie der Herstellung weltweiter Märkte hat auch Einzug in die Entwicklungspolitik der EU gehalten. So hatte die Europäische Kommission beim jüngsten EU-Afrika-Gipfel die anwesenden afrikanischen Vertreter dazu aufgefordert, insbesondere auch untereinander Handelsschranken zu beseitigen, um dadurch für mehr Wachstum und Dynamisierung zu sorgen. ${ }^{2}$

Auch wenn die EU sowohl eine Binnenliberalisierung als auch eine Liberalisierung des Welthandels ausdrücklich begrüßt und mit ihrer Politik unmissverständlich zu einer wirtschaftlichen Globalisierung beiträgt, betont sie zugleich in den letzten Jahren, dass mit der Globalisierung auch negative Effekte verbunden sein können, insofern die aus der Globalisierung entstandenen Vorteile ungleich auf verschiedene Länder und auf verschiedene Bevölkerungsgruppen in diesen Ländern verteilt sein können. Mögliche negative Effekte sollen durch einen fairen Welthandel und starke internationale Institutionen und Regulierungen abgemildert werden. Entsprechend wurde von der Kommission ein Arbeitsprogramm mit dem Titel „Programm für nachhaltige Entwicklung“ verabschiedet. Darin heißt es unter anderem: „Der Globalisierungsprozess hat für viele Menschen in der ganzen Welt beträchtliche Fortschritte mit sich gebracht. Ohne weltweit wirksame politische Entscheidungsstrukturen führt das derzeitige Globalisierungsmodell jedoch zu unausgewogenen Ergebnissen, weshalb es einer nachhaltigen globalen Entwicklung nicht zuträglich sein dürfte. Nicht alle Länder und Regionen profitieren gleichermaßen von der Globalisierung." (Kommission der Europäischen Gemeinschaften 2004: 1). Die EU müsse daher „,...] auch ihre außenpolitische Tätigkeit so gestalten, dass die Globalisierung allen Gesellschaftsgruppen in allen Partnerländern und Regionen einen optimalen Nutzen bringt" (Kommission der Europäischen Gemeinschaften 2004: 2). Um von der Globalisierung negativ betroffene Arbeitnehmer innerhalb der EU zu stützen, wurde im Jahr 2005 der so genannte „Europäische Globalisierungsfonds“ (EGF) als Anpassungshilfe und Zeichen der Solidarität eingerichtet. Dieser mit jährlich 500 Millionen Euro ausgestattete Fonds soll Arbeitnehmer, die „ernsthaft und persönlich betroffen sind von durch Änderungen im Handelsgefüge bedingter Arbeitslosigkeit" (Kommission der Europäischen Gemeinschaften 2005: 4) die Wiedereingliederung in den Arbeitsmarkt erleichtern: unter anderem sind Umschulungen, Mobilitätsbeihilfen, Hilfe bei der Stellensuche und Berufsberatung vorgesehen. Die Bemühungen der EU, die möglichen negativen Effekte einer liberalisierten globalisierten Wirtschaft abzufedern, stellen aber in keiner Weise die Grundphilosophie des Wirtschaftsskriptes der EU in Frage; die sozialen Maßnahmen sind ergänzende, vom Volumen her betrachtet eher harmlose Interventionen.

Fassen wir die Ergebnisse zusammen: Die EU verfolgt mit ihren Wirtschaftsvorstellungen vor allem ein zentrales „Megaziel“. Sie möchte Wachstum, Fortschritt und

2 Vgl. Kommission der Europäischen Gemeinschaften 2007b, Kapitel 3; ebenfalls EurActiv: ,EUAfrika-Gipfel bei Handelsfragen erfolglos', 10.12.2007. 
Prosperität für alle Bürger der Mitgliedsländer, dann aber auch für alle Weltbürger erzeugen. Dieses Ziel sieht die EU am besten erreichbar, wenn Wettbewerb, Marktbedingungen und die Liberalisierung wettbewerbsbehindernder Reglementierungen institutionalisiert werden. Dabei transportiert sie ihre Vorstellungen und Erfahrungen, die im Kontext der Herstellung eines europäischen Wirtschaftsraumes entwickelt wurden, auf die Weltgesellschaft insgesamt. Die Liberalisierung der globalen Märkte bringt Vorteile für alle beteiligten Akteure, so der Grundtenor des Wirtschaftsskripts der EU. Ob diese Sichtweise von den Bürgerinnen und Bürgern der EU geteilt wird, werden wir im folgenden Kapitel untersuchen.

Im Vordergrund des Globalisierungsskripts der EU steht die Wirtschaft. Daneben hat die EU aber auch bezüglich anderer Politikfelder Globalisierungsvorstellungen entwickelt, die wir zumindest kurz erwähnen wollen.

1. Umweltschutz: Die Europäische Union betreibt seit den 70er Jahren des letzten Jahrhunderts eine aktive Umweltpolitik (Barnes und Barnes 1999; Bailey 2003; Knill 2003; Gerhards und Lengfeld 2008) und hat dieses Engagement gerade im Kontext der Klimaschutzpolitik zunehmend globalisiert (Vogler 1999; Anderson et al. 2007). ${ }^{3}$ Sie hat wesentlich dazu beigetragen, dass 1997 in Kyoto ein Protokoll zum Schutz des Klimas verabschiedet werden konnte. Der Schutz der Umwelt ist auch als eines von zwei Schlüsselprioritäten des Arbeitsprogramms für nachhaltige Entwicklung festgeschrieben. Neben der Sicherstellung eines hohen Schutzmaßes der natürlichen Ressourcen stehen die Reduktion von Umweltverschmutzung einerseits und ein nachhaltiger Energieverbrauch zur Förderung eines ökologischen Wirtschaftswachstums andererseits im Fokus der Anstrengungen (Rat der Europäischen Union 2006: $3)$.

2. Politik: Die Europäische Union hat Vorstellungen über die Rolle und die Inhalte von Politik im Zeitalter der Globalisierung entwickelt. Diese Vorstellungen beziehen sich zum einen (a) auf Strukturmerkmale der Organisation von Politik, zum anderen (b) auf die inhaltliche Ausgestaltung des Politischen.

2.1 Die EU versteht sich als regional integriertes politisches System, welches als Vorbild für andere Länder bzw. Weltregionen dienen kann (Fligstein und Merand 2001: 26). Als Freihandelszone und Zollunion gewann sie Vorbildwirkung und gab den Anstoß für andere Formen regionaler Integration wie die NAFTA, die APEC und Mercosur (Laffan 1998: 238). Dementsprechend scheint die Europäische Kommission eine weltpolitische Landkarte $\mathrm{zu}$ favorisieren, welche sich durch stark integrierte Regionen kennzeichnet, zwischen welchen eine institutionalisierte und auf rechtlichen Grundlagen fußende Kooperation stattfindet. ${ }^{4}$ Folglich hat die EU zahl-

3 Siehe Interview mit José Manuel Barroso vom 5.12.2007, Klimawandel und Energie ,ganz oben auf der EU-Agenda', siehe Euractiv.

4 Folgendes Zitat unterstreicht diese Sichtweise: “The new globalized order should be advanced through the leadership of a 'G8'-style collection of regional organizations, of which the EU would 
reiche bilaterale regionale Kooperationsmechanismen hervorgebracht und hält in regelmäßigen Abständen Treffen mit den Hauptpartnern ab, was unter dem Label „new regionalism“ Eingang in die politikwissenschaftliche Diskussion gefunden hat (Eriksen 2006: 261). Darüber hinaus setzt sich die EU für starke internationale Organisationen ein. ${ }^{5}$

2.2 Die EU spricht sich auf globaler Ebene mit Nachdruck für Demokratie, Menschenrechte, und Rechtsstaatlichkeit als Elemente einer "good governance“ aus. Tanja Börzel und Thomas Risse (2004) haben genau analysiert, wie die Modellvorstellungen der EU diesbezüglich aussehen. Sie beschreiben, wie sich das Demokratieskript der EU im Zeitverlauf entwickelt hat und mit welchen Maßnahmen dieses in Richtung Entwicklungsländer und in Richtung Beitrittsländer der EU exportiert als spezifisch europäisches Modell profiliert wurde. ${ }^{6}$

\section{Die Einstellung der EU-Bürgerinnen und Bürger zu den Globalisierungsvorstel- lungen der EU}

Die EU ist, wie wir im letzten Kapitel gesehen haben, ein entscheidender Befürworter einer ökonomischen Globalisierung und verspricht sich durch eine Öffnung der Märkte Wachstum und Wohlstand für alle Bürger. Ob dieser Globalisierungsoptimismus von den Bürgern geteilt wird, ist eine empirisch offene Frage. Wir gehen von der Vermutung aus, dass die Bürger den Prozess der ökonomischen Globalisierung eher skeptisch bewerten und die ablehnenden Einstellungen die positiven überwiegen und dies aus folgendem Grund.

Die Diskussion der Folgen der Globalisierung beherrscht die wissenschaftliche und politische Debatte der letzten 10 Jahre. Täglich finden sich Nachrichten, die innerstaatliche und internationale Themen und Problemlagen mit Prozessen der ökonomischen Globalisierung in Verbindung bringen. Dabei werden einerseits die Vorteile und Chancen diskutiert, die mit einer Öffnung der Märkte verbunden sein können; die Thematisierung möglicher negativer Folgen scheinen nach unserem Eindruck aber die Debatte zu majorisieren. Von einigen Analysten wird betont, dass unter Bedingungen zunehmender Globalisierung bestimmte Sektoren der einheimi-

be one." Guy Verhofstadt 2001, seinerzeit amtierender EU-Ratspräsident: open letter: the paradoxes of anti-globalization (http:// premier.fgov.be/tpoics/press/e_press23.html).

5 Ein Exempel ist zum Beispiel das Engagement der EU für die Errichtung des Internationalen Strafgerichtshofs.

6 The EU has a responsibility to help 'set globalization within a moral framework' (European Council 2001). Annex 1: 'Laeken Declaration on the future of the European Union', Presidency Conclusions, Laeken Meeting of the European Council, 14_/15 Dec. Press Release: Laeken*/Brussels (14.12.01) No. 00300/1/01 (http://ue.eu.int/Newsroom/related.asp?max_/1\&bid_/76\&grp_/4061\&lang_/1). 
schen Wirtschaft nicht mehr wettbewerbsfähig sind, weil ausländische Unternehmungen die hergestellten Produkte auf der Basis wesentlich geringerer Löhne preiswerter herstellen und anbieten können. Dies gilt vor allem für die Sektoren, die einfache und standardisierte Güter herstellen und diese mit einer gering qualifizierten Belegschaft produzieren. Die Folge einer nachlassenden Konkurrenzfähigkeit ist eine Zunahme von Konkursen, die Abwanderung von Unternehmen ins Ausland und ein erhöhter Druck auf die Technisierung und Rationalisierung der Produktion. All dies führt zu einer Erhöhung der Arbeitslosigkeit vor allem unter den gering qualifizierten Erwerbspersonen und zu einem stagnierenden oder degressiven Nettoeinkommen der Beschäftigten (Altvater/Mahnkopf 1999). Begleitet wird dieser Prozess durch eine Schwächung der Verhandlungsmacht der Gewerkschaften, bedingt durch die Erhöhung der „Austrittsmöglichkeiten des Kapitals" (Streeck 1998: 186). Zugleich erhöht sich durch diesen Prozess die Ungewissheit im Hinblick auf die Sicherheit des eigenen Arbeitsplatzes.

Ein weiterer Diskussionsstrang in der Debatte über die Folgen von ökonomischen Globalisierungsprozessen bezieht sich auf das veränderte Verhältnis von Wirtschaft und Staat (vgl. zusammenfassend Beck 1997; Zürn 1998). Manche Autoren gehen davon aus, dass die Wirtschaft zunehmend aus der gesellschaftlichen und vor allem der politischen Kontrolle „entbettet“ wird (Altvater und Mahnkop 1999: 116 ff), die Märkte der Politik davonlaufen (Habermas 1998) und damit dem Staat zentrale Ressourcen und Kontrollmöglichkeiten entzogen werden. Wenn mit einer Zunahme der Globalisierung auch eine Zunahme der Arbeitslosigkeit verbunden ist, dann mindert dies zugleich die Steuereinnahmen des Staats und damit seine Handlungsfähigkeit. Offene Grenzen ermöglichen es den Unternehmungen zudem, in so genannte Steueroasen abzuwandern oder zumindest eine Abwanderung wegen zu hoher einheimischer Steuersätze anzudrohen, um damit eine Senkung der Unternehmenssteuer im eigenen Land zu erwirken (Zürn 1998; Habermas 1998). Auch dies hat negative Auswirkungen auf die Steuereinnahmen des Staates. Die dadurch mit ausgelöste Krise der öffentlichen Haushalte führt zu einem Abbau des Sozialstaates und erhöht die Ungleichheit innerhalb der Gesellschaft (Habermas 1998a), so zumindest ein häufig in der Debatte formuliertes Argument.

$\mathrm{Ob}$ sich nun diese und andere Argumente, die den Prozess der ökonomischen Globalisierung eher skeptisch beurteilen, in den Köpfen der Bürger verfangen und sie zu einer eher globalisierungsskeptischen Einstellung motivieren oder ob die Bürger den globalisierungsoptimistischen Kurs der EU unterstützen, wollen wir im folgenden prüfen. Als Datenbasis für unsere Analyse greifen wir auf die Eurobarometer-Umfrage Flash EB 151b „Globalisation“ zurück. Die Umfrage wurde vom 8.-16. Oktober 2003 vom Institut Gallup-Europe im Auftrag der Generaldirektion Presse und Kommunikation der Europäischen Kommission durchgeführt. Insgesamt wurden 7.515 Personen ab einem Alter von 15 Jahren in Form von face-to-face-Interviews befragt. Die erreichte Sample-Größe je Land variiert zwischen 498 Personen in Belgien und 507 im Vereinigten Königreich. Weil die Umfrage im Jahr 2003, also noch 
vor der EU-Erweiterung 2004 durchgeführt wurde, beschränkt sich unsere Analyse auf die "alten“ EU-15-Mitgliedsstaaten. Eine Untersuchung, welche auch die nach 2003 der EU beigetretenen Länder vollständig beinhalten würde, wäre sicherlich von Interesse, ist aber nicht möglich, weil die entsprechenden Daten fehlen. Der ausgewählte Datensatz ist der einzige, der die persönliche Zustimmung zur Globalisierung direkt abfragt. Andere Eurobarometer-Umfragen enthalten lediglich einzelne Fragen nach den Auswirkungen der Globalisierung oder der Rolle der EU in diesem Prozess. Der verwendete Fragebogen enthält darüber hinaus einige weitere Fragen zum Thema Globalisierung, welche wir für die Erklärung von Globalisierungseinstellungen benutzen können. ${ }^{7}$

Die Einstellung der Bürger zur ökonomischen Globalisierung lässt sich mit folgender Frage sehr gut operationalisieren: „Globalisation is the general opening-up of all economies, which leads to the creation of a truly world-wide market. Are you personally totally in favour, rather in favour, rather opposed or totally opposed to the development of globalisation?" Die der eigentlichen Frage vorangestellte Erläuterung richtet den Fokus des Verständnisses von Globalisierung eindeutig auf die wirtschaftlichen Aspekte dieser Entwicklung und insbesondere auf die Schaffung eines weltweiten Marktes und entspricht damit sehr genau den Vorstellungen der EU im Hinblick auf eine ökonomische Globalisierung.

Schaubild 1 fasst die Zustimmungen der Bürger in 15 europäischen Ländern zu einer ökonomischen Globalisierung zusammen. Rund 62\% der befragten EU-Bürger unterstützen die Entwicklung einer ökonomischen Globalisierung. Von 62,45\% sind ein Anteil von 47,95\% ,eher dafür“ und 14,5\% „,absolut dafür". Auf der anderen Seite sind von den $28,36 \%$ der Befragten, welche den Globalisierungsprozess tendenziell negativ bewerten, lediglich 8,01\% „absolut gegen“ eine ökonomische Globalisierung. Bis auf Griechenland gibt es in allen Ländern eine Mehrheit von Personen, welche dem Prozess der Globalisierung positiv gegenüber eingestellt sind. Insgesamt gilt also, dass die Mehrheit der Bürger eine Öffnung der Märkte unterstützt. Bedenkt man, mit welcher Skepsis ökonomische Globalisierungsprozesse in der massenmedialen Öffentlichkeit beobachtet und kommentiert werden und in welchem Maße negative Effekte der Globalisierung - Arbeitsplatzverluste, Zunahme sozialer Ungleichheit, Verlust von nationaler Identität durch Zunahme von Migration, Kapital- und Steuerflucht in Niedriglohnländer oder Steueroasen - fokussiert werden, dann ist es schon

7 Da es sich bei dem verwendeten Datensatz um eine Flash-Eurobarometer-Umfrage handelt, müssen wir Einschränkungen hinsichtlich der abgefragten soziodemographischen Merkmale hinnehmen. So wurde z.B. nicht wie sonst üblich die politische Links-Rechts-Orientierung abgefragt, eine Dimension, welche in Bezug auf die Einstellung gegenüber Globalisierung sicherlich von Relevanz sein könnte. 
überraschend, dass fast 2/3 der Bürger eine ökonomische Globalisierung eher befürworten als ablehnen. ${ }^{8}$

Zugleich fallen die Unterstützungsraten in den einzelnen Ländern recht unterschiedlich aus. Dabei haben die Bürgerinnen und Bürger der Niederlande $(77,8 \% \mathrm{Zu}-$ stimmung), Deutschlands, Finnlands, Italiens, Portugals und Irlands $(67-70 \% \mathrm{Zu}-$ stimmung) die positivste Einstellung zur Globalisierung. Insbesondere die Niederlande zeichnen sich durch einen Anteil von 23,2\% der Befragten aus, welche die Entwicklung der Globalisierung absolut befürworten. Griechenland ist das einzige Land, in welchem die Meinung der Bevölkerung mit 47,4\% Zustimmung gegenüber $50,8 \%$ Ablehnung mehrheitlich negativ gegenüber der Globalisierung eingestellt ist. Spanien (50,1\%) und Österreich (50\%) zeichnen sich ebenfalls durch eine vergleichsweise geringere Anzahl von Globalisierungsunterstützern aus. ${ }^{9}$

8 Es gibt nur wenige andere vergleichbare Untersuchungen über die Einstellungen zur Globalisierung (vgl. Stokes 2001; Scheve und Slaughter 2006: 219). Eine Übersicht über die in der EU durchgeführten Meinungsumfragen zum Thema Globalisierung bietet der Bericht von Ward und Morley (2008). Auch darin bestätigt sich der Eindruck, wonach in fast allen EU-Ländern ein tendenziell positives Bild von Globalisierung besteht. In einer repräsentativen Umfrage des „Chicago Council on Global Affairs" aus dem Jahr 2007 gaben z.B. 51\% der Franzosen - welche sich im Rahmen der Studie als am globalisierungskritischsten zeigten - an, die Globalisierung sei eher gut für ihr Land, wohingegen $41 \%$ der Befragten dieser Meinung widersprachen (Chicago Council on Global Affairs 2007: 1). Gleichwohl zeigt diese Studie auch auf, dass die hohe allgemeine Zustimmung zur Globalisierung schnell schwindet, sobald konkrete Auswirkungen und Befürchtungen thematisiert werden. Auch in den USA findet der Globalisierungsprozess in der Bevölkerung eine mehrheitliche Zustimmung (Program on International Policy Attitudes 2004).

9 Spanien und Portugal weisen mit rund 19\% den größten Anteil von Personen auf, welche auf die Frage nach der Einstellung gegenüber der Globalisierung keine Antwort geben konnten oder wollten. 
Schaubild 1: Zustimmung zur ökonomischen Globalisierung in 15 Ländern der EU (in \%)

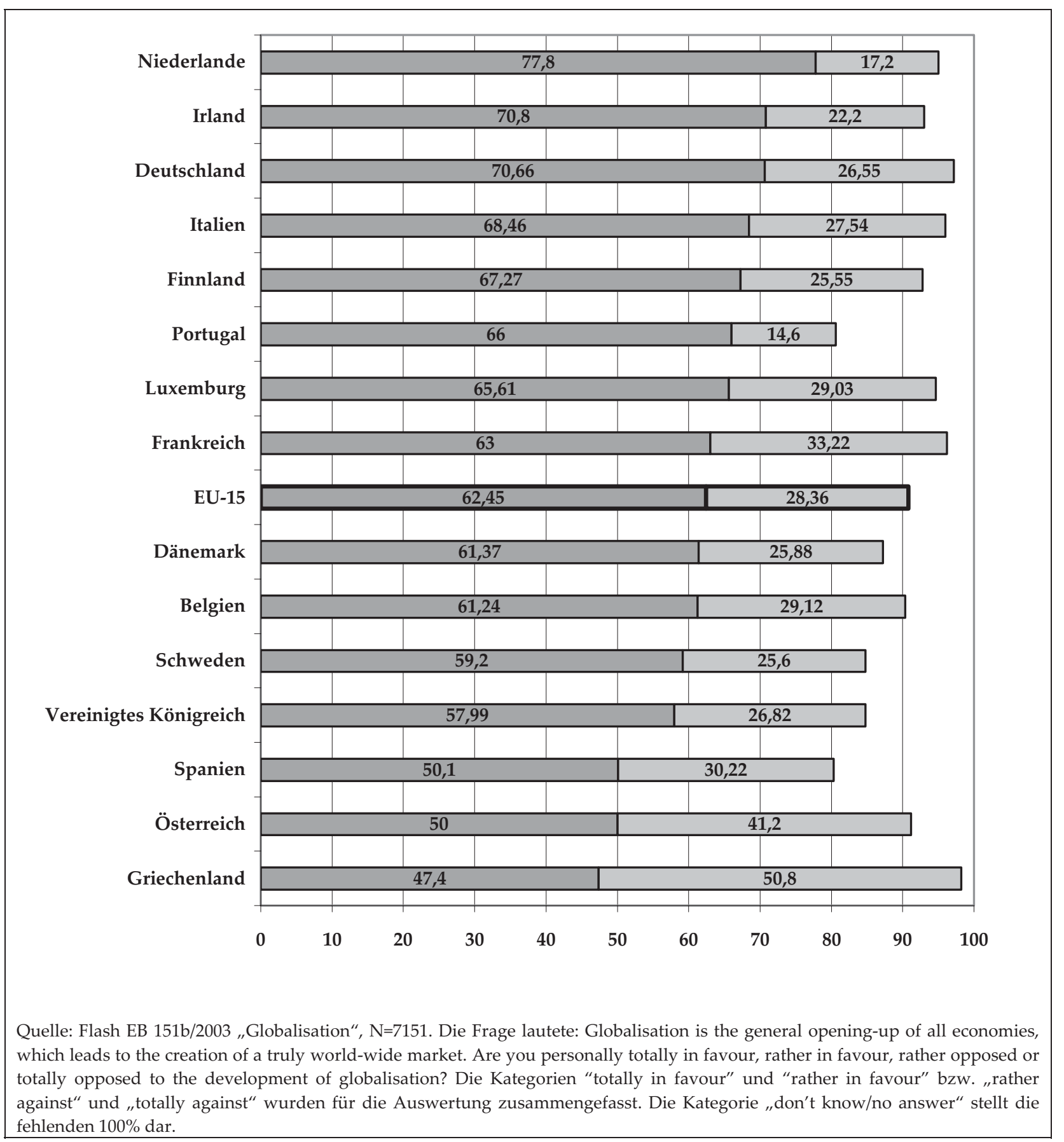

Versucht man die Länderunterschiede zu interpretieren, so findet sich auf den ersten Blick kein klar erkennbares Strukturmuster: Weder gruppieren sich die Länder in ihrer Unterstützung für den Globalisierungsprozess nach reichen und vergleichsweise ärmeren EU-Ländern, noch lässt sich erkennen, dass Wohlfahrtsstaatsregime einen Einfluss auf die Zustimmungsraten haben, noch scheint die Größe eines Landes und damit die Abhängigkeit vom Welthandel einen Einfluss auf den Globalisierungsoptimismus zu haben. 


\section{Gewinner und Verlierer: Hypothesen zur Erklärung der Unterschiede in den Einstellungen zur Globalisierung}

Wir hatten im letzten Kapitel gesehen, dass die Einstellungen zur ökonomischen Globalisierung zwischen den Ländern und zwischen den Individuen innerhalb eines Landes zum Teil beträchtlich variieren. Wir widmen das folgende Kapitel der Frage, wie man diese Unterschiede erklären kann.

Dabei greifen wir auf ein allgemeines und zugleich einfaches Theorem zurück, das wir durch drei Gruppen von Bestimmungsfaktoren weiter spezifizieren. Wir gehen davon aus, dass diejenigen, die durch Globalisierungsprozesse benachteiligt werden, sich eher gegen eine ökonomische Globalisierung aussprechen als diejenigen, für die dies nicht gilt. „Benachteiligung“ kann sich auf drei unterschiedlichen Ebenen manifestieren, die wie drei konzentrische Kreise einen jeweils unterschiedlichen Radius haben.

Den engeren Kreis bilden die subjektiven Einschätzungen der Befragten, in welchem Maße der Prozess der wirtschaftlichen Globalisierung ihnen Nachteile bzw. Vorteile bringen wird. Dabei unterscheiden wir zwischen der Einschätzung der individuellen und der volkswirtschaftlichen Vor- und Nachteile. Um diese Bestimmungsfaktoren theoretisch zu begründen, stützen wir uns in erster Linie auf das von Martin Fishbein und Icek Ajzen entwickelte Wert-Erwartungs-Modell (value-expectancy-model), auf das wir im nächsten Abschnitt noch eingehen werden (Fishbein 1963; Fishbein und Ajzen 1975). Den zweiten Kreis bildet die objektive Lage des Befragten, die dieser in der Sozialstruktur seines Landes einnimmt. Wir gehen davon aus, dass mit bestimmten Lagen im Hinblick auf den ökonomischen Globalisierungsprozess eher Vorteile, mit anderen eher Nachteile verbunden sind. Wir werden entsprechend prüfen, in welchem Maße die Berufsposition und die Bildung des Befragten die Einstellungen zur Globalisierung beeinflussen. Schließlich haben Individuen nicht nur subjektive Einschätzungen von Sachverhalten, sind nicht nur Positionsinhaber innerhalb einer Sozialstruktur, sondern sind eingebettet in die objektive Lage des Landes, in dem sie leben. Wir vermuten, dass die Makrokontexte einer Gesellschaft ebenfalls die Einstellungen zur Globalisierung beeinflussen und gehen davon aus, dass eine hohe Arbeitslosigkeit und eine geringe Marktöffnung eines Landes zu einer verstärkten $\mathrm{Ab}$ lehnung von Globalisierungsprozessen führen et vice versa. Die drei Theoreme werden im Folgenden Schritt für Schritt erläutert.

\subsection{Subjektiv erwarteter Globalisierungsnutzen}

Zur Begründung unserer ersten Hypothese nehmen wir Bezug auf die WertErwartungs-Theorie, wie sie in den 1960er Jahren von dem amerikanischen Sozialpsychologen Martin Fishbein (1963) eingeführt und dann in Zusammenarbeit mit Icek Ajzen zur „Theory of Planned Behaviour“ weiterentwickelt wurde. Die Theorie 
von Fishbein und Ajzen möchte an sich keine Einstellungen, sondern die Handlungen von Menschen erklären. Das allgemeine Modell der Handlungserklärung sieht folgendermaßen aus: Eine Handlung einer Person wird am stärksten von der Handlungsintention der Person determiniert. Die Handlungsintention wiederum wird auf zwei Dimensionen zurückgeführt: auf eine Einstellungskomponente und eine soziale Normkomponente. Personen intendieren, diejenige Handlung auszuführen, die sie (a) selbst positiv bewerten, und von der sie (b) glauben, dass für sie bedeutsame Personen es positiv bewerten würden, wenn sie dieses Verhalten zeigen würden. Da wir Einstellungen von Befragten (in unserem Fall zur Globalisierung) erklären wollen, benötigen wir aus der Theorie von Fishbein und Ajzen nur den Teil aus der Kausalkette, der sich mit den Faktoren beschäftigt, die wiederum die Einstellungen erklären können.

Gemäß dem Wert-Erwartungs-Modell hängt die Einstellung einer Person gegenüber einem spezifischen Einstellungsobjekt wie z.B. „Globalisierung“ von dem positiven oder negativem Wert ab, den sie mit dem Einstellungsobjekt verbindet und der subjektiven Wahrscheinlichkeit, mit der der jeweilige Wert eintritt. Je stärker negativ bewertete Attribute und je schwächer positiv bewertete Attribute mit einem bestimmten Einstellungsobjekt assoziiert werden, desto negativer ist auch die Einstellung gegenüber diesem Objekt. Die Einstellung gegenüber einem Einstellungsobjekt besteht also aus einer einfachen Produktsumme der Einstellungen (subjektive Wahrscheinlichkeiten, dass das Objekt $X$ das Merkmal $Y$ besitzt) und den Bewertungen dieser Merkmale. Bezeichnet man diese Produktsumme als "Nettonutzen“, so lässt sich zusammenfassen: Je größer der subjektiv erwartete Nettonutzen eines Einstellungsobjektes ist, desto positiver ist die Einstellung gegenüber diesem Objekt.

Da die subjektive Zuschreibung von Merkmalen zu bestimmten Einstellungsobjekten in keiner Weise den objektiven Tatsachen entsprechen muss, eignet sich dieses Modell besonders gut, um Einstellungen gegenüber komplexen Objekten zu erklären, hinsichtlich derer eine Person nur über unvollständiges Wissen verfügt und entsprechend nur annäherungsweise die tatsächlichen Auswirkungen eines Prozesses wie z.B. der Globalisierung einschätzen kann. Übertragen auf unseren Untersuchungsgegenstand erwarten wir auf der Grundlage des Wert-Erwartungs-Modells, dass ein größerer "Nettonutzen“ der Globalisierung mit einer positiveren Bewertung dieser einhergeht. Der von uns ausgewertete Datensatz ermöglicht es, zwei Dimensionen von Nettonutzen zu spezifizieren.

a) Die Befragten wurden zum einen gefragt, ob sie glauben, dass eine weitere Globalisierung für sie und die eigene Familie mehr Vorteile oder mehr Nachteile bringen wird. Wir bezeichnen diese Dimension als erwarteten individuellen Globalisierungsnutzen.

b) Die Interviewten wurden weiterhin gefragt, ob sie glauben, dass 1. Globalisierung für die Firmen ihres Landes eine gute Gelegenheit und 2. dass Globalisierung eine Bedrohung der Beschäftigung darstelle. Wir haben aus diesen beiden Fragen ei- 
nen additiven Index (siehe Anhang) gebildet und bezeichnen diese Dimension als volkswirtschaftlichen Globalisierungsnutzen.

\subsection{Sozialstrukturelle Lage des Befragten}

Die Werterwartungstheorie steht im Widerspruch zu den Annahmen des klassischen Rational-Choice-Ansatzes, der nicht den subjektiven Einschätzungen von Nutzen, sondern dem objektiven Nutzen die stärkste Erklärungskraft zubilligt. Die realen Vor- und Nachteile von Individuen ergeben sich häufig aus deren Positionierung innerhalb der Sozialstruktur ihrer Gesellschaft. Von dieser Vermutung gehen wir auch im Hinblick auf die Einstellungen zur Globalisierung aus. Entscheidend scheinen hier vor allem das Bildungsniveau sowie die berufliche Stellung eines Individuums zu sein (Scheve und Slaughter 2006).

In der Literatur wird die Auffassung vertreten, dass die Vorteile, welche aus der zunehmenden Marktliberalisierung als Folge der ökonomischen Globalisierung entstehen, asymmetrisch unter den Bewohnern eines Landes verteilt sind. Personen mit geringem Bildungsgrad und niedriger beruflicher Stellung werden von Globalisierungsprozessen weniger Vor- als Nachteile haben (Gabel 1998: 43-44) und dies aus folgenden Gründen. Parallel zum Globalisierungsprozess hat die Nachfrage an höher qualifizierten Arbeitskräften zu ungunsten der Nachfrage nach schlechter ausgebildeten Arbeitskräften zugenommen (Slaughter und Swagl 1997: 2). Diese Entwicklung wird von einer Steigerung der Arbeitslosigkeitsraten von schlechter ausgebildeten Arbeitskräften begleitet, wobei zwischen Ländern mit flexiblen und eher rigiden Arbeitsmärkten zu unterscheiden ist: In Ländern mit flexiblen Arbeitsregelungen, wie z.B. in den USA, hat dies zu einer Steigerung der Lohnspreizung zwischen besser und schlechter ausgebildeten Arbeitskräften geführt. In Ländern mit rigiden Arbeitsmärkten, wie z.B. Frankreich und Deutschland, resultierte dies hingegen in höheren Arbeitslosigkeitsraten für schlechter ausgebildete Arbeitskräfte (Slaughter und Swagl 1997: 4). Auch gibt es Anzeichen dafür, dass die Reallöhne von schlechter ausgebildeten Arbeitskräften von der Auslagerung vor allem von arbeitsintensiver Produktion (Outsourcing) negativ beeinflusst werden, wohingegen höher ausgebildete Arbeitskräfte von diesem Prozess profitieren (Geishecker und Görg 2004). Es ist weiterhin zu erwarten, dass Kapitalbesitzer überproportional durch die Ausweitung von Anlagemöglichkeiten von der Liberalisierung des Kapitalmarkts profitieren. EUBürger mit vergleichsweise niedrigen Löhnen werden durch eine Kapitalmarktliberalisierung benachteiligt, da ihre Wohlfahrt primär von den Löhnen und Gehältern sowie staatlichen Transferzahlungen abhängt, welche durch die Liberalisierung zunehmend gefährdet werden (Frieden 1991: 434). 
All diese Faktoren lassen die Vermutung zu, dass schlechter ausgebildete Arbeitskräfte zu den Verlierern der Globalisierung gehören. ${ }^{10} \mathrm{Zu}$ den Gewinnern scheinen vor allem hoch ausgebildete Arbeitskräfte sowie Kapitalbesitzer zu gehören (Sinn 2004: 118). Wir operationalisieren die sozialstrukturelle Position eines Befragten mit Hilfe von zwei Variablen: Der Bildung des Befragten (gemessen durch das Alter, in dem er oder sie die Ausbildung beendet hat) und die Berufsposition des Befragten, wobei die „manuell Tätigen“ die Referenzkategorie darstellen (siehe Anhang).

Die hier vermutete Wirkungsweise von Bildung und Berufsposition auf Globalisierungseinstellungen mag aber je nach Kontext, indem ein Individuum platziert ist, unterschiedlich ausfallen. Mit Bezug auf die Arbeit von O'Rourke (2003) vermuten wir, dass in reichen Ländern ein hohes Ausbildungsniveau und eine hohe berufliche Stellung einen positiven Einfluss auf die Einstellung gegenüber Globalisierung haben, in ärmeren Ländern sollte dagegen ein gegenteiliger Effekt vorzufinden sein. O'Rourke erläutert diesen Zusammenhang unter Rückgriff auf das Heckscher-OhlinTheorem beispielhaft für den Fall einer Wirtschaft mit lediglich zwei Gütern, in welcher sich die Länder ausschließlich durch ihre Ausstattung mit qualifizierten Arbeitskräften unterscheiden. Die verhältnismäßigen bzw. relativen Löhne sind, unter der ceteris paribus-Annahme, in Ländern mit einem hohen Qualifikationsniveau bzw. reichen Ländern niedriger als in Ländern mit geringerem Qualifikationsniveaus und einer höheren Gewichtung des Produktionsfaktors Arbeit. Dies führt dazu, dass die reichen Länder unter Ausnutzung ihres komparativen Kostenvorteils wissensintensive Güter herstellen und exportieren werden, wogegen ärmere Länder weniger wissensintensive und eher arbeitsintensive Güter herstellen werden. Werden nun Handelsschranken abgebaut, führt dies zu einer Angleichung der Faktorpreise: So steigt der relative Lohn wissensintensiver Produktion in reichen Ländern, wogegen er in ärmeren Ländern fällt. Die relativ häufigeren Produktionsfaktoren gewinnen so an realen Werten, während die selteneren an Wert verlieren. Gut ausgebildete Personen sollten daher in reichen Ländern Globalisierung im Sinne einer Marktöffnung unterstützen, wohingegen sie in ärmeren Ländern Globalisierung ablehnen sollten (O'Rourke 2003: 6). Ob Bildung und Berufsposition in reichen und in armen Ländern einen unterschiedlichen Einfluss auf die Einstellungen zur Globalisierung haben, prüfen wir, indem wir in der multivariaten Analyse eine Interaktionsvariable gebildet aus der Bildung des Befragten und dem Bruttoinlandsprodukt pro Kopf berücksichtigen.

10 Für den erwarteten Effekt von Bildung auf die Einstellung zur Globalisierung mag es noch eine zusätzliche Begründung geben. Ronald Inglehart (1977) geht davon aus, dass erhöhte Bildung zu einer erhöhten kognitiven Mobilisierung führt, welche ein Individuum in der Lage versetzt, politische Prozesse auf einem gewissen Abstraktionsniveau zu verfolgen und diese in Beziehung zu den eigenen Wertorientierungen sowie der personellen Lage zu setzen. Dieser Wirkungsmechanismus mag auch für die Beurteilung des Prozesses der Globalisierung von Relevanz sein, der durch ein hohes Maß an Abstraktion und Komplexität gekennzeichnet ist (vgl. auch Janssen 1991). 


\subsection{Makroökonomische Situation des Landes}

Auch die makroökonomische Situation eines Landes, in dem ein Befragter lebt, sollte unserer Annahme nach einen Einfluss auf seine Bewertung der Globalisierung ausüben, wobei insbesondere zwei Bestimmungsmerkmale in der Literatur diskutiert werden.

1. Wir gehen davon aus, dass die Arbeitslosigkeitsrate eines Landes einen Einfluss auf die individuelle Bewertung der Globalisierung hat. Wie wir bereits ausgeführt haben, besteht Grund zu der Annahme, dass der Prozess der Globalisierung vor allem in Ländern mit rigiden Arbeitsmärkten mit einer Steigerung der Arbeitslosigkeitsrate insbesondere für schlechter ausgebildete Arbeitskräfte einhergeht. Hohe Arbeitslosigkeitsraten sind dabei nicht nur negativ für die betroffenen Personen, sondern - durch steigende Ausgaben für die sozialen Sicherungssysteme sowie einen Verlust an Produktionsleistung - auch für die gesamte Volkswirtschaft und tangieren damit mittelbar alle Bürger. Die Arbeitslosigkeitsrate ist darüber hinaus ein maßgeblicher Wirtschaftsindikator. Sie wird in den Medien vielfach zitiert und ebenso häufig mit Globalisierungsprozessen in einen ursächlichen Zusammenhang gebracht (Slaughter und Swagl 1997: 4). Wir erwarten deshalb, dass in Ländern mit einer hohen Arbeitslosenrate die Zustimmung zur Globalisierung geringer ausfällt als in Ländern mit niedriger Arbeitslosenquote. Wir haben entsprechend die Arbeitslosenrate zum Zeitpunkt der Erhebung der Eurobarometerumfrage (2003) in der multivariaten Analyse berücksichtigt.

2. Die ökonomische Offenheit eines Landes im Sinne der Integration einer Volkswirtschaft in den Weltmarkt durch Handel und Kapitalströme sollte ebenfalls einen Einfluss auf die individuelle Globalisierungsbewertung haben. In welche Richtung dieser Einfluss wirkt, ist aber in der Literatur umstritten. Die Integration in den Weltmarkt beeinflusst Länder in vielerlei Hinsicht. Zunächst einmal profitieren Länder von den Gewinnen aus dem Außenhandel, was ein wichtiger Anreiz für den Aufbau von Handelsbeziehungen mit anderen Ländern ist und zur weiteren ökonomischen Globalisierung beiträgt (Koster 2007: 4). Weiterhin beeinflusst die Integration in den Weltmarkt die Politik von Regierungen, insbesondere bezüglich wohlfahrtsstaatlicher Sicherungsprogramme. So kann wirtschaftliche Offenheit zu einer Spaltung zwischen gut ausgebildeten und weniger gut ausgebildeten Arbeitern führen, da letztere durch die gestiegene Mobilität von Produktionsfaktoren eher um den Verlust ihres Arbeitsplatzes fürchten müssen. Eine hohe Abhängigkeit vom Außenhandel kann weiterhin zu einer Unterminierung von nationalen Normen und sozialen Institutionen führen, da Regierungen nicht mehr in der Lage sind, soziale Sicherung in ausreichendem Maße zu gewährleisten (Koster 2007: 4). An anderer Stelle wurde in diesem Zusammenhang argumentiert, dass viele Personen ein höheres Maß an Unsicherheit aufgrund von wirtschaftlicher Offenheit wahrnehmen (Blossfeld et al. 2006; Scheve und Slaughter 2004). Die Frage, ob die Offenheit einer Volkswirt- 
schaft eher mit Vor- oder mit Nachteilen für die Bürger verbunden ist, ist also eine in der Literatur offene Frage. Wir haben entsprechend diesbezüglich keine gerichtete Hypothese formuliert. Die ökonomische Offenheit eines Landes haben wir in der multivariaten Analyse durch den so genannten „KOF-Globalisierungsindex“ operationalisiert, der verschiedene Indikatoren über die ökonomische und soziale Offenheit eines Landes enthält (siehe Anhang).

\section{Empirische Prüfung der Hypothesen}

Die von uns im letzten Kapitel begründeten unabhängigen Variablen lagern auf zwei unterschiedlichen Ebenen, auf der der befragten Individuen und auf der Ebene der Makrokontexte der Länder (Arbeitslosenrate und Offenheit der Volkswirtschaft). Um dieser Datenstruktur methodisch gerecht zu werden, ist eine Mehrebenenanalyse an sich das angemessene Verfahren. Diese haben wir in einem ersten Schritt auch durchgeführt. Die Ergebnisse, die hier nicht separat ausgewiesen werden, zeigen, dass der Einfluss der beiden Kontextvariablen nicht signifikant ist. Auch die Erklärungskraft des Modells erhöht sich nicht, wenn man zusätzlich zu den Individualvariablen die beiden Kontextvariablen berücksichtigt. Wir beschränken uns deswegen im Folgenden auf die Darstellung der Ergebnisse der einfachen Regressionsanalysen, die für den Leser auch besser nachzuvollziehen ist.

Zur Prüfung unserer Hypothesen haben wir insgesamt drei Regressionsmodelle berechnet (Tabelle 1). Die genaue Beschreibung der Variablen zur Operationalisierung der theoretischen Konstrukte befindet sich in Anhang 1. Das Modell 1 überprüft den Einfluss des subjektiv erwarteten persönlichen sowie volkswirtschaftlichen Globalisierungsnutzens auf die Einstellung zur Globalisierung. Modell 2 überprüft, ob die beiden sozialstrukturellen Individualmerkmale - berufliche Stellung und Bildungsniveau - einen eigenständigen Effekt auf die Globalisierungseinstellung besitzen. Da wir davon ausgehen, dass die Wirkungsweise dieser beiden Faktoren je nach Wohlstand des Landes variiert, haben wir zusätzlich eine Interaktionsvariable (gebildet aus dem Bruttoinlandsprodukt pro Kopf des Landes und der Bildung des Befragten) in der Analyse berücksichtigt. Modell 3 enthält dann zusätzlich die beiden Makrovariablen. 
Tabelle 1: Erklärung der Einstellung zur Globalisierung: Lineare Regressionen

\begin{tabular}{|c|c|c|c|}
\hline & Modell 1 & Modell 2 & Modell 3 \\
\hline $\begin{array}{l}\text { Individueller erwar- } \\
\text { teter Globalisie- } \\
\text { rungsnutzen }\end{array}$ & $\begin{array}{c}.275 \\
(18.35)^{* *}\end{array}$ & & $\begin{array}{c}.271 \\
(18.06)^{* *}\end{array}$ \\
\hline $\begin{array}{l}\text { Erwarteter volks- } \\
\text { wirtschaftlicher } \\
\text { Globalisierungs- } \\
\text { nutzen }\end{array}$ & $\begin{array}{c}.288 \\
(19.19)^{* *}\end{array}$ & & $\begin{array}{c}.290 \\
(19.23)^{* *}\end{array}$ \\
\hline Bildung & & $\begin{array}{c}-.138 \\
(-3.48)^{*}\end{array}$ & $\begin{array}{l}-.069 \\
(-1.65)\end{array}$ \\
\hline Selbstständig & & $\begin{array}{r}.018 \\
(1.09) \\
\end{array}$ & $\begin{array}{c}.018 \\
(1.28) \\
\end{array}$ \\
\hline Angestellt & & $\begin{array}{c}.045 \\
(2.24)^{*} \\
\end{array}$ & $\begin{array}{c}.041 \\
(2.35)^{*} \\
\end{array}$ \\
\hline Nicht berufstätig & & $\begin{array}{c}.039 \\
(1.95) \\
\end{array}$ & $\begin{array}{c}.033 \\
(1.93) \\
\end{array}$ \\
\hline Bildung*BIP & & $\begin{array}{c}.114 \\
(2.86)^{*} \\
\end{array}$ & $\begin{array}{c}.018 \\
(0.42) \\
\end{array}$ \\
\hline Arbeitslosenrate & & & $\begin{array}{c}-.024 \\
(-1.37)\end{array}$ \\
\hline $\begin{array}{l}\text { Ökonomische Of- } \\
\text { fenheit }\end{array}$ & & & $\begin{array}{c}.023 \\
(1.32) \\
\end{array}$ \\
\hline $\mathbf{R}^{2}$ & 0.2509 & 0.0034 & 0.2561 \\
\hline $\mathrm{N}$ & 4979 & 4979 & 4979 \\
\hline
\end{tabular}

Quelle: Flash Eurobarometer 151b „Globalisation“, Oktober 2003.

1. Lineare Regressionen, standardisierte Koeffizienten, t-Werte in Klammern, Gewichtet nach Stichprobengröße, ${ }^{*} \mathrm{p}<, 05 .{ }^{* *}$ $\mathrm{p}<, 01$.

2. Die abhängige Variable hat, wie oben erläutert, vier Ausprägungen „,totally in favour“, „rather in favour“, „rather opposed“ und „totally opposed“. Die Merkmalsausprägung „weder noch“ wurde für die Regressionsanalysen als fehlender Wert (missing) definiert.

3. Die Berufskategorie „Arbeiter“ bildet die Referenzkategorie.

Blicken wir zunächst auf unser Ausgangsmodell (Modell 1) in der zweiten Spalte von Tabelle 1. Wie vermutet, haben die subjektiven Nutzenerwartungen einen stark positiven und signifikanten Effekt auf die Einstellung zum Globalisierungsprozess. Je stärker Befragte glauben, dass eine weitere Globalisierung für sie und die eigene Familie Vorteile bringen wird und je stärker sie glauben, dass auch die eigene Volkswirtschaft von der Globalisierung profitieren wird, desto höher ist der antizipierte Nutzen und desto stärker fällt die Unterstützung für den Globalisierungsprozess aus. Die Erklärungsmächtigkeit der beiden Variablen ist mit einer Varianzaufklärung von rund $25 \%$ sehr hoch.

Modell 2 prüft, in welchem Maße die sozialstrukturellen Variablen Berufsstatus und Bildungsniveau einen Einfluss auf die Globalisierungseinstellungen haben. Die Erklärungskraft der beiden Variablen ist, wie man an dem $\mathrm{R}^{2}$ sieht, vernachlässigend 
gering. Von den Berufskategorien sind lediglich die "Angestellten“ etwas globalisierungsfreundlicher eingestellt als die Arbeiter. Der Effekt ist aber so gering, dass er kaum zu interpretieren ist. Das Bildungsniveau des Befragten, gemessen am Alter bei Beendigung der Vollzeitbildung, besitzt - entgegen der Erwartung, dass höher gebildete Personen eine positivere Einstellung zur Globalisierung besitzen - einen negativen Einfluss auf die individuelle Globalisierungseinstellung. Die Tatsache, dass die Interaktionsvariable - gebildet aus dem Bruttoinlandsprodukt pro Kopf sowie dem Bildungsniveau - einen signifikant positiven Effekt auf die Einstellung zur Globalisierung besitzt, scheint die Annahme des Heckscher-Ohlin-Theorems zu stützen, dass Bildung und Berufsposition in reichen und in armen Ländern einen unterschiedlichen Einfluss auf die Einstellungen zur Globalisierung haben können.

In Modell 3 haben wir zusätzlich die beiden makroökonomischen Kontextvariablen berücksichtigt. Wie in der Mehrebenenanalyse zeigt sich auch hier, dass weder die Arbeitslosenrate noch der Grad der ökonomischen Offenheit eines Landes einen signifikanten Einfluss auf die individuelle Globalisierungseinstellung besitzen. Zudem werden die Bildungsvariable und die Interaktionsvariable in ihrem Effekt auf die Globalisierungseinstellung insignifikant, so dass, betrachtet man das Gesamtmodell, nur die individuellen Nutzenerwartungen die Einstellung zur Globalisierung erklären können.

Bilanzieren wir unsere Befunde mit Rückgriff auf unsere Hypothesen: Einstellungen zur Globalisierung werden nahezu ausschließlich durch die subjektiven Nutzenerwartungen der Bürger beeinflusst. Erwartet ein Befragter einen positiven Nutzen von der Globalisierung für sich sowie die heimische Volkswirtschaft, so geht dies mit einer positiven Bewertung der Globalisierung einher; dieses Ergebnis stützt unsere erste Hypothese, die wir aus der Wert-Erwartungs-Theorie abgeleitet haben. Die Vermutung, dass schlechter ausgebildete Personen und Personen mit einem niedrigen sozialen Status, die potentiell zu den Verlierern der Globalisierung gehören, Globalisierungsbestrebungen eher ablehnen, kann nicht bestätigt werden. Und auch die Kontextfaktoren der Länder, Arbeitslosenrate und Offenheit der Volkswirtschaft, haben entgegen der theoretischen Erwartung keinen Einfluss auf die Globalisierungseinstellung der Bürger.

Wir haben unsere empirischen Analysen durch eine zusätzliche Auswertung ergänzt. Die Werterwartungstheorie legt nahe, dass die drei Variablengruppen in einer Kausalkette hintereinander gelagert sind, so dass die subjektiven Nutzenerwartungen die Globalisierungseinstellungen beeinflussen, sie aber wiederum durch die sozialstrukturellen Merkmale der Befragten beeinflusst werden. Wir haben entsprechend geprüft, in welchem Maße die Bildung und die Berufsposition des Befragten nicht nur die Globalisierungseinstellungen beeinflussen, sondern den subjektiv erwarteten persönlichen Globalisierungsnutzen und den erwarteten volkswirtschaftli- 
chen Globalisierungsnutzen. Die Ergebnisse der Analyse sind im folgenden Pfaddiagramm dargestellt. ${ }^{11}$

Schaubild 2: Einfluss der soziodemographischen Individualmerkmale auf die subjektiven Nutzenerwartungen

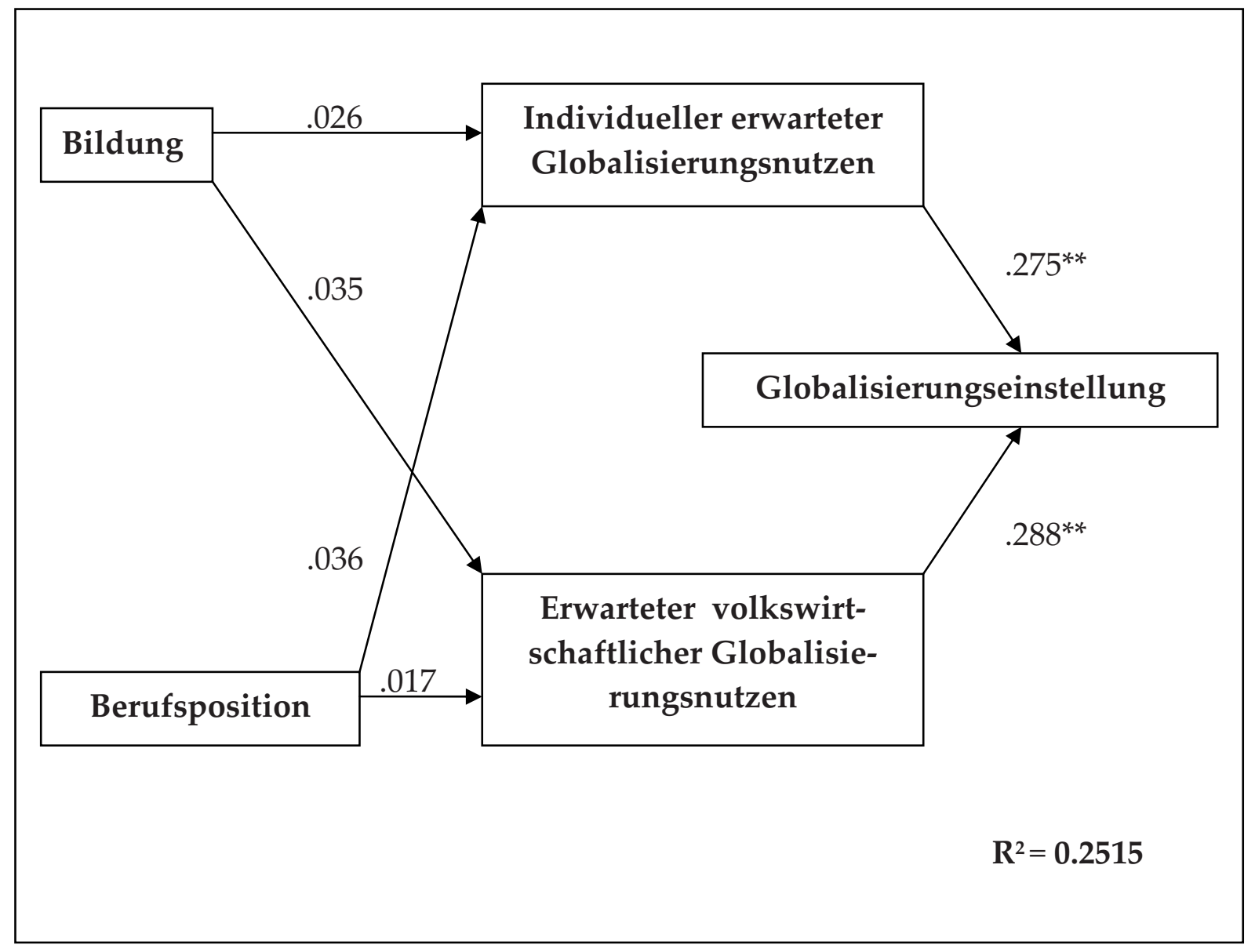

Die Analysen zeigen, dass Bildung und Berufsposition keinen signifikanten Effekt auf die Nutzenerwartungen der Bürger haben. Es bleibt damit bei dem Befund, dass die Globalisierungseinstellungen der Bürger allein von deren Nutzenerwartungen bestimmt werden, ohne dass diese selbst wiederum sozialstrukturell angebunden sind.

\section{Zusammenfassung}

Auf der Basis einer Interpretation von Gesetzestexten und öffentlichen Verlautbarungen haben wir in einem ersten Schritt die Vorstellungen der Europäischen Union von einer globalisierten Wirtschaft rekonstruiert. Die EU verfolgt mit ihren Wirt-

${ }^{11}$ Die Berufsposition wurde der besseren Übersicht halber für diesen Zweck dichotimisiert (,alle Berufsgruppen“ versus "Arbeiter"). 
schaftsvorstellungen und deren Implementierung durch konkrete Politiken das Ziel, Wachstum, Fortschritt und Prosperität für alle Bürger der Mitgliedsländer und für alle Weltbürger zu erzeugen. Dieses Ziel sieht die EU am besten erreichbar, wenn Wettbewerb, Marktbedingungen und die Liberalisierung wettbewerbsbehindernder Reglementierungen institutionalisiert werden. Dabei transportiert sie ihre Vorstellungen und Erfahrungen, die im Kontext der Herstellung eines europäischen Wirtschaftsraumes entwickelt wurden, auf die Weltgesellschaft insgesamt. Die Liberalisierung der globalen Märkte bringt Vorteile für alle beteiligten Akteure, so die Grundannahme des Wirtschaftsskripts der EU.

$\mathrm{Ob}$ diese Sichtweise von den Bürgerinnen und Bürgern der EU geteilt wird, haben wir im zweiten Schritt auf der Grundlage einer Auswertung des Eurobarometers für 15 Länder der EU geprüft. Das Ergebnis ist überraschend: 63\% der befragten EUBürger unterstützen die Entwicklung einer ökonomischen Globalisierung. Bis auf Griechenland gibt es in allen Ländern eine Mehrheit von Personen, die dem Prozess der Globalisierung positiv gegenüber eingestellt sind. Angesichts der Skepsis, mit der ökonomische Globalisierungsprozesse in der massenmedialen Öffentlichkeit kommentiert werden, ist es erstaunlich, dass fast 2/3 der Bürger eine weitere ökonomische Globalisierung im Sinne einer Marktliberalisierung befürworten. Zugleich fallen die Unterstützungsraten in den einzelnen Ländern recht unterschiedlich aus. Während die Bürgerinnen und Bürger der Niederlande zu 77,8\% eine positive Einstellung zur ökonomischen Globalisierung an den Tag legen, sind es in Griechenland nur 47,4\%. Und auch die länderinternen Streuungen sind zum Teil erheblich. Zur Erklärung dieser Unterschiede sind wir von der Annahme ausgegangen, dass diejenigen, die durch Globalisierungsprozesse benachteiligt werden, sich eher gegen den Globalisierungsprozess aussprechen als diejenigen, für die dies nicht gilt. „Benachteiligung" kann sich in drei Dimensionen manifestieren: In der subjektiven Interpretation von positiven und negativen Folgen von Globalisierungsprozessen, in den Nachteilen, die sich aus der objektiven sozialstrukturellen Lage des Individuums ergeben und aus den Nachteilen, die aus der ökonomischen Situation des Landes resultieren.

Auch unsere Kausalanalyse kann mit einem überraschenden Befund aufwarten. Zwar können die Einstellungen der Bürger zur Globalisierung recht gut erklären, allerdings spielen die für Soziologen im Zentrum stehenden Variablen dabei keine bzw. fast keine Rolle. Weder die makroökonomische Situation des Landes, noch die sozialstrukturelle Lage des Interviewten haben einen nennenswerten Effekt auf deren Globalisierungseinstellungen. Die Haltung zur Globalisierung wird fast ausschließlich bestimmt durch den subjektiv definierten Globalisierungsnutzen; dieser ist selbst nicht mehr rückgekoppelt an die objektive Lage des Individuums oder des Landes, in dem das Individuum lebt. Nicht die „objektive“ Nutzenkalkulation, die man aus der Struktur des Landes und der Position des Individuums in der Sozial- 
struktur ableiten kann, bestimmen die Einstellungen zur Globalisierung, sondern allein die durch das Individuum definierten Nutzenerwägungen.

Demnach scheinen Personen unabhängig von ihrer objektiven Situation den Globalisierungsprozess vor allem dann zu unterstützen wenn sie subjektiv der Meinung sind, dass dieser von Nutzen für sie und die heimische Volkswirtschaft ist. Nun kann man die von den Befragten definierten Situationsdefinitionen nicht einfach nur als reine subjektive Konstruktionen abtun. Spätestens seit dem berühmten und vielfach zitiertem Theorem von William und Dorothy Thomas (1928: 572) "if men define situations as real, they are real in their consequences", wissen wir, dass den individuellen Situationsdefinitionen und damit auch den Nutzendefinitionen eine reale Qualität zukommt, insofern sie die Handlungen von Menschen anleiten können.

Die subjektive Zuschreibung von Merkmalen zu bestimmten Einstellungsobjekten ist aber besonders für sehr komplexe Einstellungsobjekte und für lebensweltlich ferne Themen von besonderer Relevanz. Bei solchen Themen können Menschen die tatsächlichen Auswirkungen nicht gut einschätzen. Die Merkmale „Komplexität des Themas" und „Lebensweltferne“ scheinen für das Globalisierungsthema zuzutreffen. Es handelt sich um ein überaus komplexes Themengebiet; die realen Vor- und Nachteile, die mit Globalisierungsprozessen verbunden sein können, sind selbst unter Experten überaus umstritten. Und auch der Bezug zur alltäglichen Lebenswelt der Menschen ist nicht an und für sich gegeben. Ob z.B. die eigene Arbeitslosigkeit in irgendeiner Weise kausal mit der ökonomischen Globalisierung in Verbindung zu bringen ist, ist eine offene Frage, und hängt entscheidend von kausalen Attributionsprozessen ab. Diese Ausgangskonstellation macht es möglich, dass sich die Einstellungen auch von der objektiven Lage der Sozialposition des Individuums und der des Landes entkoppeln können. Die Beeinflussbarkeit durch eine öffentliche Neudefinition der Situationen ist entsprechend hoch. Für deutungsoffene Themen gilt aber, dass den Massenmedien eine besondere Bedeutung in der Interpretation der Themen zukommt. Insofern könnte man schlussfolgern, dass die Europäische Union das aus ihrem Blickwinkel Richtige tut, wenn sie Broschüren und Verlautbarungen mit Titeln wie „Globalisierung als Chance für alle“ oder "Globalisierung als Chance nutzen“ herausgibt, um die Akzeptanz für ihr Globalisierungsskript zu erhöhen. 


\section{Literaturverzeichnis}

Altvater, E., Mahnkopf, B. (1999): Grenzen der Globalisierung. Ökonomie, Ökologie und Politik in der Weltgesellschaft. Münster: Westfälisches Dampfboot.

Anderson, J., Bowyer, C., Fergusson, M., Pallemaerts, M., Valsecchi, C. (2007): Background Paper on European Actions on Climate Change and Energy. Institute for European Environmental Policy, Bailey, I. (2003): New Environmental Policy Instruments in the European Union. Aldershot: Ashgate.

Barnes, P.M., Barnes, I.G. (1999): Environmental Policy in the European Union. Cheltenham etc.: Elgar.

Blossfeld, H.P., Buchholz, S., Hofäcker, D. (2006): Globalization, Uncertainty, and Late Careers in Society. London: Routledge.

Börzel, T., Risse, T. (2004): One Size Fits All! EU Policies for the Promotion of Human Rights, Democracy and the Rule of Law, Workshop on Democracy Promotion, Oct. 4-5 2004, Center for Development, Democracy, and the Rule of Law, Stanford University, http://iisdb.stanford.edu/pubs/20747/Risse-Borzel-stanford_final.pdf (27.12.07).

Chicago Council on Global Affairs (2007): World Public Favors Globalization and Trade but Wants to Protect Environment and

Jobs, http://www.worldpublicopinion.org/pipa/articles/btglobalizationtradera/349.php?lb=btgl\&pnt= $349 \&$ nid $=\& i d=(28.12 .07)$.

Dreher, A. (2006): 'Does Globalization Affect Growth? Evidence from a new Index of Globalization', Applied Economics 38(10): 1091-1110 (s. Dreher, A., Gaston, N. and Martens, P. (2008): Measuring Globalization - Gauging its Consequences. New York: Springer.)

Eriksen, E.O. (2006): The EU - a cosmopolitan polity? Journal of European Public Policy 13(2): 252-69.

EurActiv: ,EU-Afrika-Gipfel bei Handelsfragen erfolglos' (10.12.07), http://www.euractiv.com/de/handel/eu-afrika-gipfel-handelsfragen-erfolglos/article-168993 (28.12.07).

EurActiv: Barroso, J.M. (2007): ,Klimawandel und Energie ,ganz oben auf der EU-Agenda' (05.12.07), http://www.euractiv.com/de/bali/barroso-klimawandel-energie-ganz-oben-eu-agenda/article168867 (28.12.07).

EUR-Lex: ,Vertrag zur Gründung der Europäischen Gemeinschaft', Amtsblatt Nr. C 325 (24.12.02), http://eur-lex.europa.eu/de/treaties/dat/12002E/pdf/12002E_DE.pdf (27.12.07).

Europäischer Rat (2007): Schlussfolgerungen des Vorsitzes zum Europäischen Rat vom 4. Dezember 2007, Anlage II: Erklärung der EU zur Globalisierung.

Eurostat: Online Datenbank, Arbeitslosenrate für das Jahr 2003Angaben für das Jahr 2003, http://epp.eurostat.ec.europa.eu/portal/page?_pageid=1996,45323734\&_dad=portal\&_schema=P ORTAL\&screen=welcomeref\&open=/economy/na/nama/nama_gdp\&language=de\&product=EU _economy_finance\&root=EU_economy_finance\&scrollto=0 (28.12.07).

Eurostat: Online Datenbank, BIP pro Kopf in KKS für das Jahr 2003, http://epp.eurostat.ec.europa.eu/portal/page?_pageid=1996,45323734\&_dad=portal\&_schema=P ORTAL\&screen=welcomeref\&open=/\&product=STRIND_ECOBAC\&depth=2.

Fishbein, M. (1963): 'An investigation of relationships between beliefs about an object and

Fishbein, M., Ajzen, I. (1975): Belief, attitude, intention, and behavior: an introduction to theory and research. Reading, MA: Addison-Wesley Publishers.

Fligstein, N., Merand, F. (2001): Globalization or Europeanization? Evidence on the European Economy Since 1980, Conference Paper 'Shareholder Value-Capitalism and Globalization Hamburg', 10.-12.5.2001, http://repositories.cdlib.org/iir/ccop/wps-2001-02/ (07.12.07).

Frieden, J. (1991): 'Invested Interests: The Politics of National Economic Policies in a World of Global Finance', International Organization 45: 425-451. 
Gabel, M. (1998): 'Public Support for European Integration: An Empirical Test of Five Theories', The Journal of Politics 2: 333-354.

Geishecker, I., Görg, H. (2004): ,Winners and losers: Fragmentation, trade and wages revisited', Discussion Paper 385, Deutsches Institut für Wirtschaftsforschung (Hrsg.), Berlin.

Gerhards, J. (2007): Cultural Overstretch? The enlargement of the European Union and the cultural differences between old and new member states and Turkey. London und New York: Routledge.

Gerhards, J., Hölscher, M. (2003): ,Kulturelle Unterschiede zwischen Mitglieds- und Beitrittsländern der EU. Das Beispiel Familien- und Gleichberechtigungsvorstellungen', Zeitschrift für Soziologie 32: 206-225.

Gerhards, J., Lengfeld, H. (2006): ,Das Ökologieskript der Europäischen Union und seine Akzeptanz in den Mitglieds- und Beitrittsländern der EU', Zeitschrift für Soziologie 35: 24-40.

Gerhards, J., Lengfeld, H. (2008): 'Support for European Union environmental policy by citizens of EU-member and accession states', Comparative Sociology (im Erscheinen).

Habermas, J. (1998). Jenseits des Nationalstaats? Bemerkungen zu Folgeproblemen der wirtschaftlichen Globalisierung. In: Beck, Ulrich (Hrg.): Politik der Globalisierung. Frankfurt am Main: Suhrkamp. S. 67-84.

Habermas, J. (1998a) Die postnationale Konstellation und die Zukunft der Demokratie, in: Blätter für deutsche und internationale Politik, Heft 7, S. 804-817

Hay, C., Rosamond, B. (2002): 'Globalization, European integration and the discursive construction of economic imperatives', Journal of European Public Policy 9(2): 147-167.

Hölscher, M. (2006): Wirtschaftskulturen in der erweiterten EU. Die Einstellungen der Bürgerinnen und Bürger im europäischen Vergleich. Wiesbaden: VS Verlag. http://www.ieep.eu/publications/pdfs/2007/T-PAGE\%20-\%20EU\%20paper.pdf (17.03.08).

Inglehart, R. (1977): The Silent Revolution: Changing Values and Political Styles among Western European Publics. Princeton, NJ: Princeton University Press.

Janssen, J. (1991): 'Postmaterialism, Cognitive Mobilization and Public Support for European Integration', British Journal of Political Science 21(4): 443-468.

Knill, C. (2003): Europäische Umweltpolitik. Steuerungsprobleme und Regulierungsmuster im Mehrebenensystem. Opladen: Leske \& Budrich.

Kommission der Europäischen Gemeinschaften (2004): Mitteilung der Kommission an den Rat, das Europäische Parlament, den Europäischen Wirtschafts- und Sozialausschuss und den Ausschuss der Regionen, Die soziale Dimension der Globalisierung - der politische Beitrag der EU zu einer gleichmäßigen Verteilung des Nutzens, KOM(2004) 383 endgültig, Brüssel, http://ec.europa.eu/employment_social/international_cooperation/docs/ilo_com_2004_0383_de. pdf (07.12.07).

Kommission der Europäischen Gemeinschaften (2005): Europäische Werte in der globalisierten Welt. $\operatorname{KOM}(2005)$ 525, Brüssel.

Kommission der Europäischen Gemeinschaften (2006): Mitteilung der Kommission an den Rat, das Europäische Parlament, den Europäischen Wirtschafts- und Sozialausschuss und den Ausschuss der Regionen, Ein wettbewerbsfähiges Europa in einer globalen Welt - Ein Beitrag zur EU-Strategie für Wachstum und Beschäftigung. KOM(2006) 567 endgültig, Brüssel, http://eurlex.europa.eu/smartapi/cgi/sga_doc?smartapi!celexplus!prod!DocNumber\&lg=de\&type_doc=C OMfinal\&an_doc=2006\&nu_doc=567 (07.12.07).

Kommission der Europäischen Gemeinschaften (2007a): Commission Staff Working Document, The External Dimension of the Single Market Review, SEC(2007) 1519, Brüssel.

Kommission der Europäischen Gemeinschaften (2007b), First Action Plan (2008-2010) for the Implementation of the Africa-EU Strategic partnership, http://ec.europa.eu/development/icenter/repository/EAS2007_action_plan_trade_regional_integ ration_en.pdf\#zoom=100 (07.12.07). 
Kommission der Europäischen Gemeinschaften (2007c): Mitteilung der Kommission an den Rat, das Europäische Parlament, den Europäischen Wirtschafts- und Sozialausschuss und den Ausschuss der Regionen, Das europäische Interesse: Erfolg im Zeitalter der Globalisierung. KOM(2007) 581, Brüssel.

Koster, F. (2007): 'Globalization, Social Structure, and the Willingness to Help Others: a Multilevel Analysis Across 26 Countries', European Sociological Review 23(4): 537-551.

Kriesi, H., Grande, E., Lachat, R., Dolzal, M., Bornschier, S., Frey, T. (2006): 'Globalization and the transformation of the national political space: Six European countries compared', European Journal of Political Research 45: 921-956.

Laffan, B. (1998): 'The European Union: a distinctive model of internationalization', Journal of European Public Policy 5(2): 235-253.

Meunier, S., Nicolaïdis, K. (2005): The European Union as a Trade Power, http://www.princeton.edu/ amoravcs/wws556c/handover.pdf (07.12.07).

Meyer, J.W. (2000): 'Globalization: Sources and Effects on National States and Societies', International Sociology 15: 233-248.

Meyer, J.W., Boli, J., Ramirez, F.O. (1985): 'Explaining the Origins and Expansion of Mass Education', Comparative Education Review 29(2): 145-170.

Meyer, J.W., Boli, J., Thomas, G.M., Ramirez, F.O. (1997): 'World Society and the Nation State', American Journal of Sociology 103(1): 144-181.

O'Rourke, K.H. (2003): Heckscher-Ohlin Theory and Individual Attitudes Towards Globalization, Conference Paper Eli Heckscher Celebratory Symposium, Stockholm School of Economics, May 22-24, http://www.tcd.ie/Economics/TEP/2003_papers/TEPNo8KOR23.pdf (18.12.07), published in R. Findlay, R. Henriksson, H. Lindgren and M. Lundahl (Hrsg.) (2006): Eli Heckscher, International Trade, and Economic History. Cambridge, MA: MIT Press, S. 107-138.

Program on International Policy Attitudes, Center for International and Security Studies at University of Maryland (2004): Americans on Globalization, Trade, and Farm Subsidies, http://www.pipa.org/archives/us_opinion.php (28.12.07).

Rat der Europäischen Union (2006): Review of the EU Sustainable Development Strategy (EU SDS) Renewed Strategy, 10117/06, Brüssel, http://register.consilium.europa.eu/pdf/en/06/st10/st10117.en06.pdf (27.12.07).

Rosamond, B. (2002): 'Globalisation and the European Union' (Conference Paper) The European Union in International Affairs, National Europe Centre, Australian National University, 3-4 July, 2002.

Scheve, K., Slaughter, M.J. (2004): 'Economic insecurity and the globalization of production', American Journal of Political Science 48: 662-674.

Scheve, K.F., Slaughter, M.J. (2006): 'Public Opinion, International Economic Integration, and the Welfare State', in P. Bardhan, S. Bowles, M. Wallerstein (Hrsg.) Globalization and Egalitarian Redistribution. Princeton, NJ: Princeton University Press, S. 217-260.

Schild, J. (2005): ,Ein Sieg der Angst - das gescheiterte französische Verfassungsreferendum', Integration 3: 187-200.

Sinn, H.-W. (2004): ,The dilemma of globalisation: A German perspective', Économie Internationale 100(4):

111-120, http://www.cairn.info/article.php?ID_REVUE=ECOI\&ID_NUMPUBLIE=ECOI_100\&ID_ARTIC LE=ECOI_100_0111 (06.01.08).

Slaughter, M.J., Swagel, P. (1997): 'Does Globalization Lower Wages and Export Jobs?' in International Monetary Fund (Hrsg.), Economic Issues 11, http://www.imf.org/EXTERNAL/PUBS/FT/ISSUES11/INDEX.HTM (06.01.08).

Stokes, B. (2001): ,Globalisierung in der öffentlichen Meinung', Internationale Politik 7: 19-26, http://www.internationalepolitik.de/archiv/jahrgang2001/juli01/download/51317456125a11db97 99a3b1f376a013a013/original_Stokes_0107.pdf (27.12.07). 
Stone Sweet, A., Sandholz, W., Fligstein, N. (Hrsg.) (2001): The Institutionalization of Europe. Oxford: Oxford University Press.

Streeck, W. (1998): ,Industrielle Beziehungen in einer internationalisierten Wirtschaft', in U. Beck (Hrsg.) Politik der Gobalisierung. Frankfurt a.M.: Suhrkamp. S. 169-202.

Thomas, W.I., Thomas, D.S. (1928) The Child in America: Behavior Problems and Programs. New York: Knopf.

Beck, U. (1997): Was ist Globalisierung? Irrtümer des Globalismus - Antworten auf Globalisierung. Frankfurt: Suhrkamp.

Vogler, J. (1999): 'The European Union as an actor in international environmental politics', Environmental Politics 8(3): 24-48.

Ward, T., Terry, J. (2008): , Perceptions of globalisation: attitudes and responses in the $\mathrm{EU}^{\prime}$ in European Foundation for the Improvement of Living and Working Conditions (Hrsg.), http://www.eurofound.europa.eu/docs/erm/tn0708016s/tn0708016s.pdf (22.3.08).

Wobbe, T. (2001): ,Institutionalisierung von Gleichberechtigungsnormen im supranationalen Kontext: Die EU-Geschlechterpolitik' in B. Heintz (Hg.) Geschlechtersoziologie, Opladen: Westdeutscher Verlag, S. 332-355.

Wobbe, T., Biermann, I. (2007): ,Die Metamorphosen der Gleichheit in der Europäischen Union', Kölner Zeitschrift für Soziologie und Sozialpsychologie 59: 565-588.

Zürn, Michael (1998): Regieren jenseits des Nationalstaates. Globalisierung und Denationalisierung als Chance. Frankfurt: Suhrkamp. 


\section{Anhang : Beschreibung der Variablen}

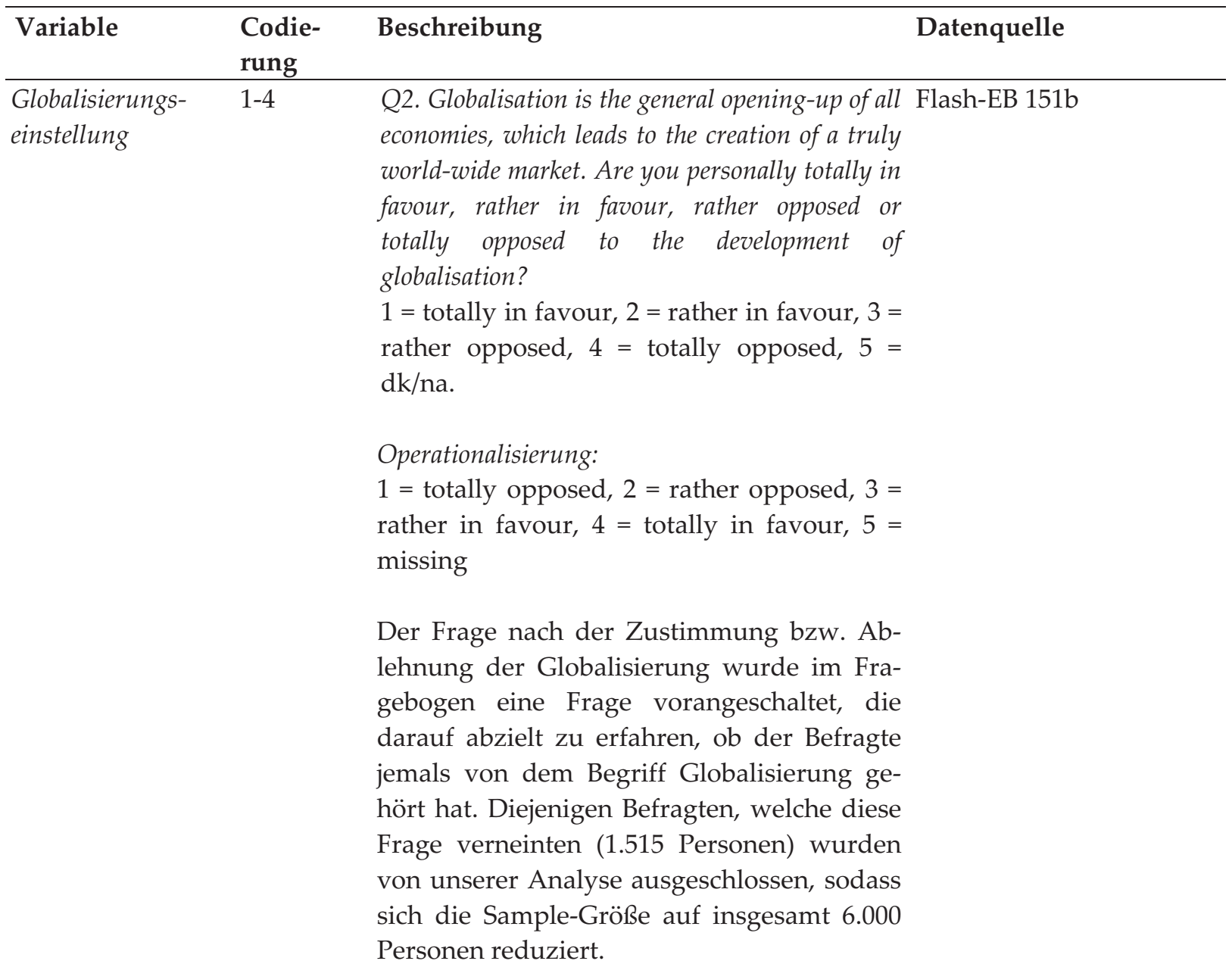

Individuell erwar- 1-3 Q8. In your opinion, if globalisation intensifies Flash-EB 151b

teter Globalisiein the future, would you say that overall this

rungsnutzen would be more or less advantageous for you and your family? (One answer possible)

$1=$ More advantageous, 2 = Less advantageous, $3=$ Neither more or less advantageous, $4=\mathrm{dk} / \mathrm{na}$.

Operationalisierung:

1 = Less advantageous, 2 = More advantageous, 3, $4=$ missing.

Index:

Erwarteter volkswirtschafticher Globalisierungsnutzen

\section{1-2}

Q6. Of the following two propositions, which is Flash-EB 151b the one which is closest to your opinion with regard to globalisation? (One Answer possible)

$1=$ Globalisation represents a good opportunity for [Nationality] 


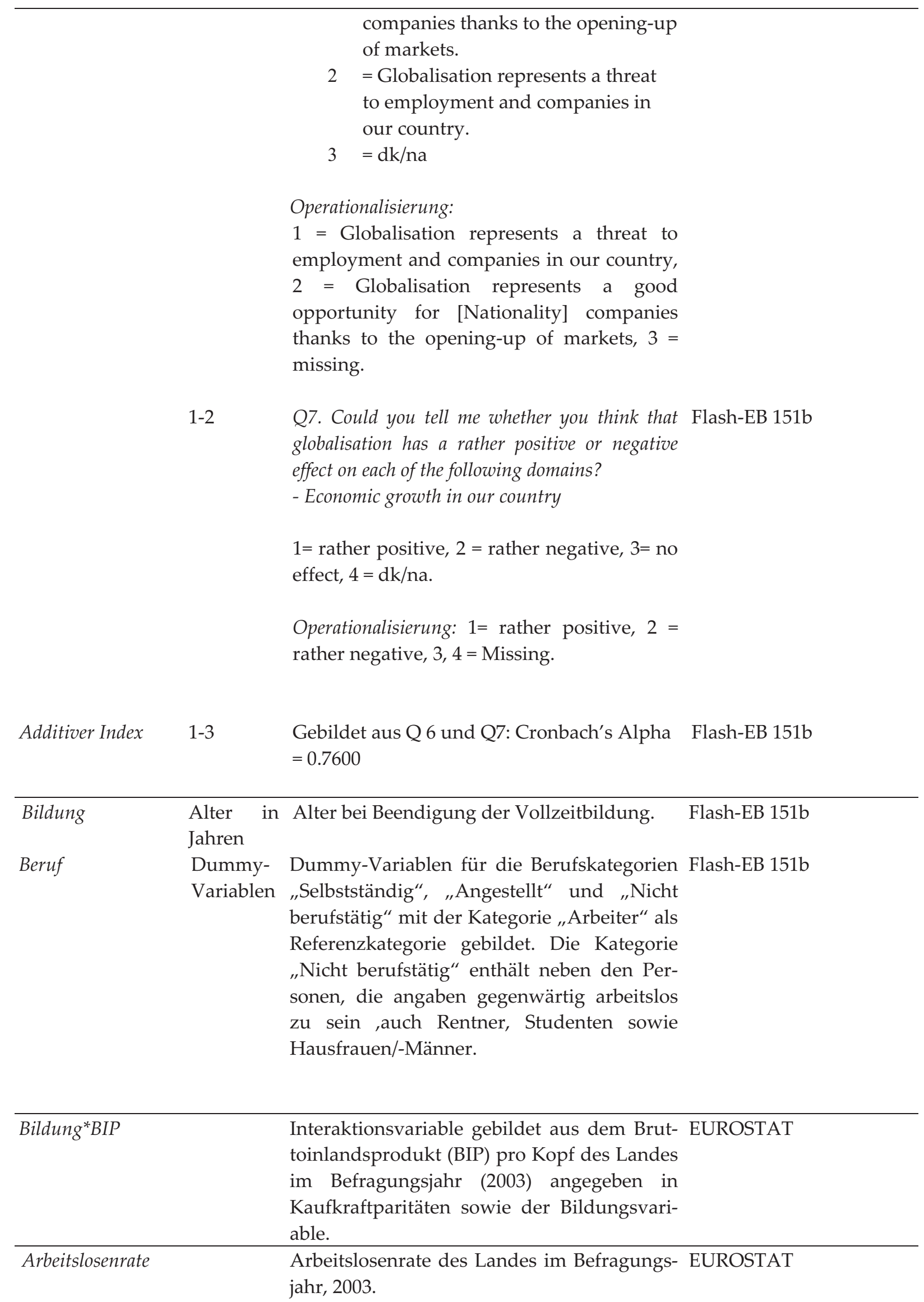


Ökonomische Globalisierung
Der KOF-Globalisierungsindikator enthält KOF-

Indikatoren über ökonomische und soziale Globalisierungsindikator Offenheit zahlreicher Länder seit dem Jahr

1970. Jede der beiden Offenheitsdimensio- http://globalization.kof.et nen wird durch eine Skala gemessen, welche hz.ch/

von 0 (absolut geschlossen) bis 10 (absolut offen) reicht. Ökonomische Offenheit durch den Außenbeitrag des BIP's, Ausländische Direktinvestition (als Anteil am BIP), Einkommenszahlungen an Ausländer (als Anteil am BIP), versteckte Importbarrieren, die durchschnittliche Steuerrate, Zölle sowie Restriktionen bezüglich des Erwerbs von Eigentum durch ausländische Investoren operationalisiert. Soziale Offenheit beinhaltet Daten über ausgehende Telefonanrufe, Transferzahlungen (als Anteil am BIP), internationalen Tourismus, durchschnittliche Kosten eines Telefonats in die USA, Anteil der ausländischen Bevölkerung an der Gesamtbevölkerung, Telefonanschlüsse (pro 1000 Personen), Internetanschlüsse (pro Person), Internetnutzer (Anteilig zur Gesamtbevölkerung), Kabelfernsehverbindungen (pro 1000 Personen), Import und Export von Tageszeitungen sowie die Anzahl der McDonald-Restaurants in einem Land (pro Person). Die Gewichte für die verschiedenen Indices werden durch eine PrincipalComponent-Analyse ermittelt (Dreher 2006). 